(Aus der geburtshülflichen Abtheilung des Krankenhauses Charlottenburg. Leiter: Dr. C. Keller.)

\title{
Die Infection der weiblichen Harnwege durch das Bacterium coli commune in der Schwangerschaft und im Wochenbett.
}

\author{
Von \\ Dr. Paul Alsberg, \\ Assistenzarzt. \\ (Hierzul Tafel IX.)
}

Die moderne Erforschung der Harninfectionen beginnt erst mit den bakteriologischen Studien Bumm's über den puerperalen Blasenkatarrh (1886). Nachdem einmal der richtige Weg gewiesen; traten in der Folgezeit neue, concrete Werthe, die Bakterien, an die Stelle bisheriger Vermuthungen. Als einer der ersten konnte Rovsing (1889) eine gut fundirte Theorie über die Cystitisätiologie mittheilen, welche neben Tuberkelbacillen und Gonokokken nur die harnstoffzersetzenden Bakterien als Ursache der Cystitis anerkannte. Im Gegensatz zu Rovsing bezeichnete Krogius') (1891) einen sauren Harnkeim, das Bact. coli com., als den hauptsächlichsten Cystitiserreger. Damit war der Anstoss zu einer lebhaften Controverse gegeben, welche sich durch die Fortschritte in der Bakteriologie, durch die Einführung des Cystoskopes und durch die Vervollkommnung der Thierexperimente immer mehr zuspitzte, aus welcher schliesslich das Bact. coli, wenn nicht als Sieger, so doch als qualificirter Mitbewerber hervorging. Namentlich die französische Schule war es [Guyon $\left.{ }^{1}\right)$ ], welche die Bedeutung des Bact. soli für die Harnpathologie auf's eifrigste verfocht, ohne allerdings ihre Gegner vollends überzeugen zu können (Rovsing 1898). Endlich näherte sich Baisch (1904) wieder dem Rovsing'schen Standpunkte, indem er dem Bact. coli lediglich die Rolle eines

1) Cit. nach Rovsing, Baisch u. A. 

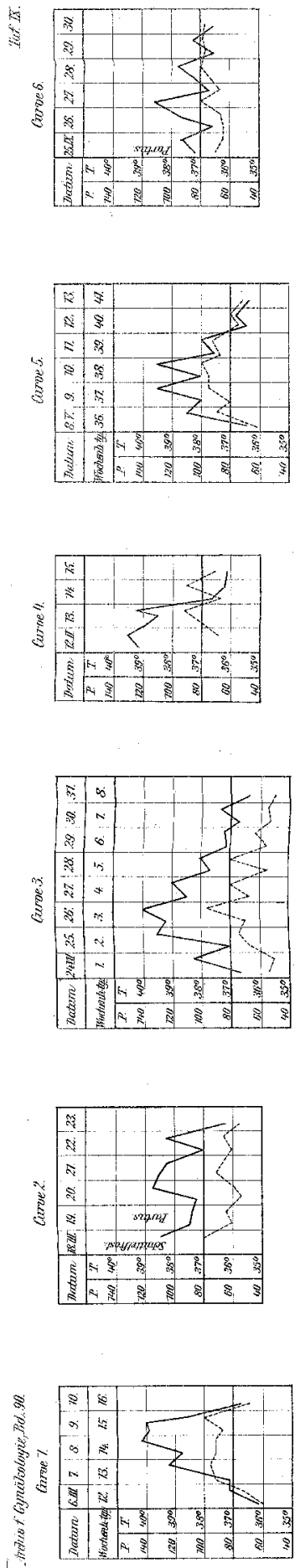
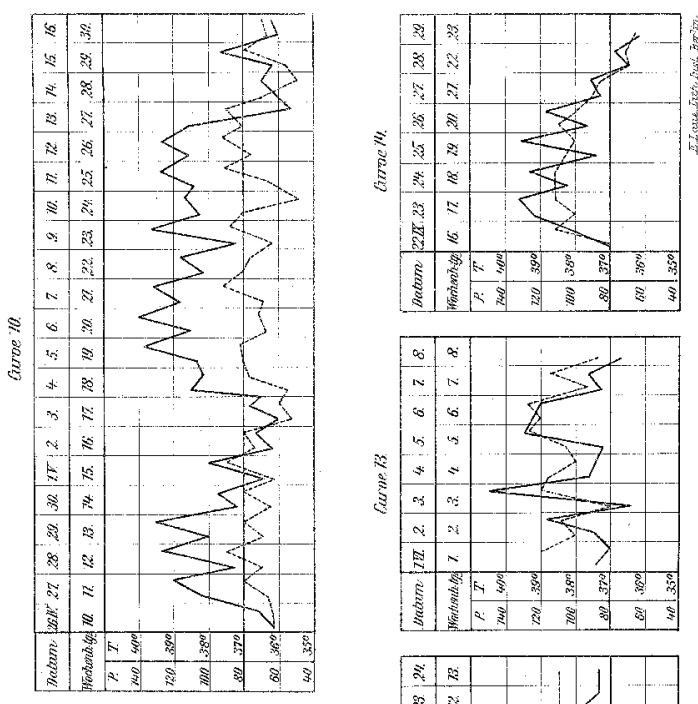

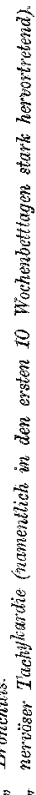
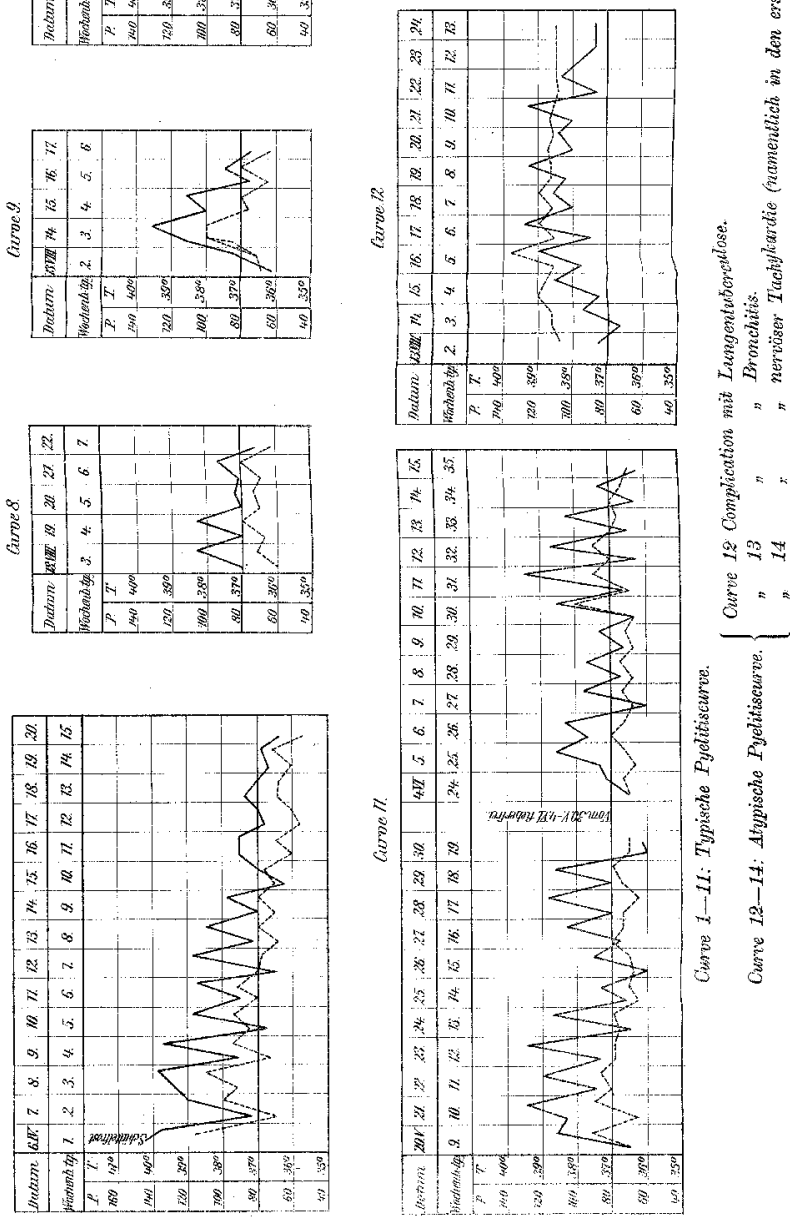
secundären Immigranten zuwies, eine Auffassung, die gleichfalls nicht unangefochten blieb (Goldberg u. A.). Wenn wir noch registriren, dass selbst im eigenen Jager der "Coli-Anhänger" über die Frage des Infectionsmodus eine neue Fehde ausbrach, die bisher keinen definitiven Austrag gefunden hat, so wird man aus dieser Skizze den Eindruck einer grossen Verworrenheit gewinnen, die noch gegenwärtig über unserem Thema liegt. Eine Erklärung für dieses Deficit mag in der Schwierigkeit einer exacten Beweisführung, in der Mannigfaltigkeit der Infectionsmöglichkeiten, in der Unzulänglichkeit der Untersuchungsmethoden und Experimente gesehen werden.

Die vorliegende Arbeit, die als ein kleiner Beitrag zu dem umfangreichen Thema aufgefasst werden soll, entstand unter dem Eindruck eines sehr hohen Procentsatzes von Coli-Harninfectionen bei unserem Material, welcher mit Macht auf die Bedeutung dieser Erkrankung hinwies und zu Untersuchungen anregte. Nothwendigerweise musste hierbei auch den übrigen Harnkeimen einige Beachtung zu Theil werden. Fernerhin war auf theoretischem Gebiete eine Abschweifung auf allgemeinere Fragen unvermeidlich. Aus alledem aber resultirte wiederum die specielle Nutzanwendung auf unser eigentliches Thema.

\section{Bakteriologische Vorbemerkungen.}

Günther giebt nach dem Vorschlage von Gilbert und Lion für das Bact. coli com. folgende Characteristica an: Eigenbewegliches Kurzstäbchen, das sowohl bei Brut- wie bei Zimmertemperatur, sowohl in Gegenwart wie bei Abwesenheit von freiem Sauerstoff zu wachsen vermag, das die Gelatine nicht verflüssigt, in seinen oberflächlichen Colonien auf der Gelatineplatte häutchenförmig wächst, Traubenzucker- sowie Milehzuckerbouillon unter Säuerung und Gasbildung vergärt und in gewöhnlicher zuckerfreier Bouillon Indol producirt. Fügen wir als weitere Eigenschaften noch die dicke gelbe Rasenbildung auf Kartoffeln, die Coagulation der Milch, die Entfärbung bei der Gram'schen Methode hinzu, so sollte man annehmen, dass eine derartige Fülle von Kriterien ein Bacterium streng zu differenciren im Stande wäre. Dem tritt aber das Bact. coli selbst entgegen durch seine grosse Launenhaftigkeit in den Lebens- und Wachsthumserscheinungen und durch die Menge von Varietäten und Spielarten, so dass man daza übergegangen ist, 
weniger von einem bestimmten constanten Bact. coli als vielmehr von einer "Coligruppe" zu sprechen. Noch in einer kürzlich erschienenen Arbeit (Burk 1907) wird eine strenge Trennung der zur Coligruppe zählenden Bakterien auf Grund morphologischer sowie biologischer Merkmale als zur Zeit noch nicht geglückt bezeichnct und darauf hingewiesen, dass in einem Darm zu gleicher Zeit immer mehrere eulturell verschiedene Colispielarten anzutreffen sind. Auch das Agglutinationsphänomen erwies sich als nicht verwerthbar (Radzievsky).

Im Verlaufe unserer Untersuchungen konnten wir uns häufig genug von dem wechselvollen Verhalten in Form, Aussehen und Lebenserscheinungen überzeugen. Neben plumpen Kurzstäbchen begegneten wir längeren, schlankeren Formen, mitunter zu Ketten angeordnet; die Eigenboweglichkeit war meist sehr ausgeprägt, nicht allzuselten aber auch sehr träge; die Säure- und Indolbildungsenergie war eine verschiedene u. s. w.

Derartige Variationen erscheinen nicht mehr verwunderlich, seitdem M. Neisser (1906) selbst in vitro Mutationsvorgänge beobachten konnte, indem er bei geeigneter Züchtung die eingebüsste Fähigkeit der Milchzuckervergährung bei dem gleichen Stamme wieder erzielte (Bact. coli mutabile). Wir möchten Pfaundler beipflichten, wenn er die überaus grosse Variationsfähigkeit des Bact. coli als einen Ausdruck seiner sonstigen saprophytischen Rolle ansieht, die eine weitgehende Anpassungsbefähigung an wechselnde Lebensbedingungen verlangt, welche ihrerseits wieder eine Reaction in Lebensäusserungen und Erscheinungsformen des Mikroben zur Folge haben muss.

Bei der Bestimmung und Beurtheilung der Bakterien leistete uns namentlich der v. Drigalski-Conradi'sche Nährboden vorzügliche Dienste, welcher - auf dem Princip der Milchzuckervergährung beruhend - durch seine intensive Röthung sofort die Anwesenheit von Bact. coli nahelegt. Färbt man einen ObjecträgerAusstrich dieser Coljcultur mit Löffler's oder polychromem Methylenblau, so erhält man neben wenigen, ganz mit Farbstoff imprägnirten Bakterien, zahlreiche oft zu mosaikartigem Rasen verinigte bläulichweisse, transparente, von einem zarten dunkleren Rande eingefasste Kurzstäbchen. Sei es, dass es sich hier um plasmolytische, um Involutions- oder sonstige Vorgänge handelt, liese durchsichtigen Bakterien erscheinen uns in diagnostischer Besiehung als äusserst charakteristisch, so dass wir die Entscheidung 
der Bakterienbestimmung - neben den anderen Kriterien - immer von der Anwesenheit dieser Formen abhängig machten ${ }^{1}$ ).

\section{Der Keimgehalt der Urethra.}

Die Bakterien der Urethra haben in Folge der naheliegenden Vermuthung ihrer Pathogenese für die Entstehung der Cystitis vielfache Beachtung gefunden. Schon Rovsing (1889) führte den Nachweis harnstoffzersetzender Keime in der Urethra. Das Bact. coli wurde zuerst von Savor (1894) in der weiblichen Harnröhre festgestellt.

In nachfolgenden Uebersichtstabellen, die sich theilweise an die Tabellen von Baisch und Piltz anlehnen, sollen die Ergeb-

Tabelle I.

1. Nichtschwangere.

\begin{tabular}{|c|c|c|c|c|c|c|c|c|c|}
\hline \multirow[b]{2}{*}{ Autor } & \multirow[b]{2}{*}{$\begin{array}{l}\text { Zahl der } \\
\text { Fälle }\end{array}$} & \multicolumn{4}{|c|}{ Vulva } & \multicolumn{4}{|c|}{ Urethra } \\
\hline & & 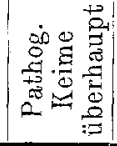 & 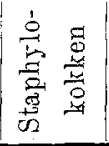 & 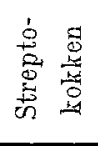 & $\begin{array}{l}\because 7 \\
8 \\
0\end{array}$ & 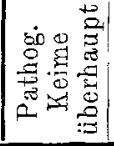 & 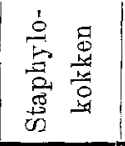 & 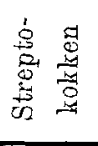 & 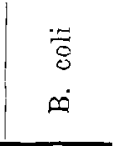 \\
\hline & & $p C t$. & $p \subset t$ & pCt. & pCt. & pCt. & pCt. & pCt. & pCt \\
\hline $\begin{array}{c}\text { y. Gawronsky } \\
(1894)\end{array}$ & 62 & - & - & - & - & $14=22$ & $9=14$ & $3=5$ & $2=3$ \\
\hline $\begin{array}{l}\text { Menge } \\
\text { (1897) }\end{array}$ & 70 & $7=10$ & $3=4$ & $2=3$ & $2=3$ & 一 & - & - & - \\
\hline $\begin{array}{l}\text { Melchior } \\
\text { (1897) }\end{array}$ & $\begin{array}{c}12 \\
\text { (speciell } \\
\text { auf Coli) }\end{array}$ & - & - & - & $6=50$ & - & - & - & - \\
\hline $\begin{array}{l}\text { Sayor } \\
(1899)\end{array}$ & 93 & - & - & - & - & $40=43$ & $22=23$ & $4=4$ & $14=15$ \\
\hline$"$ & $\begin{array}{c}12 \\
\text { (speciell } \\
\text { auf Coli) }\end{array}$ & 一 & - & - & - & $\cdots$ & - & - & $4=33$ \\
\hline $\begin{array}{c}\text { Schenk-Austerlitz } \\
\text { (1899) }\end{array}$ & 59 & - & - & 一 & - & $2=3$ & 0 & 0 & $1=1,6$ \\
\hline$(1900)$ & 25 & - & - & - & - & $1=4$ & 0 & 0 & 0 \\
\hline $\begin{array}{l}\text { Piltz } \\
(1904)\end{array}$ & 50 & $44=88$ & $33=66$ & $4=8$ & $35=70$ & $18=36$ & $17=34$ & $1=2$ & $5=10$ \\
\hline $\begin{array}{l}\text { Baisch } \\
\text { (1904) }\end{array}$ & 30 & - & - & - & - & - & $30=100$ & - & $20=66$ \\
\hline 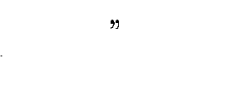 & $\begin{array}{c}45 \\
\text { (Operirte, } \\
\text { Bettlägerige) }\end{array}$ & - & - & - & - & - & - & - & $45=10 C$ \\
\hline
\end{tabular}

1) Herrn Prof. Dietrich, Prosector am Krankenbause Westend-Charlottenburg, welcher uns bei den baliteriologischen Untersuchungen freundlichst unterstützte und mebrfach die Bestimmung von Balterien äbernahm, sei an dieser Stelle bestens gedankt. 
Tabelle II.

2. Schwangere.

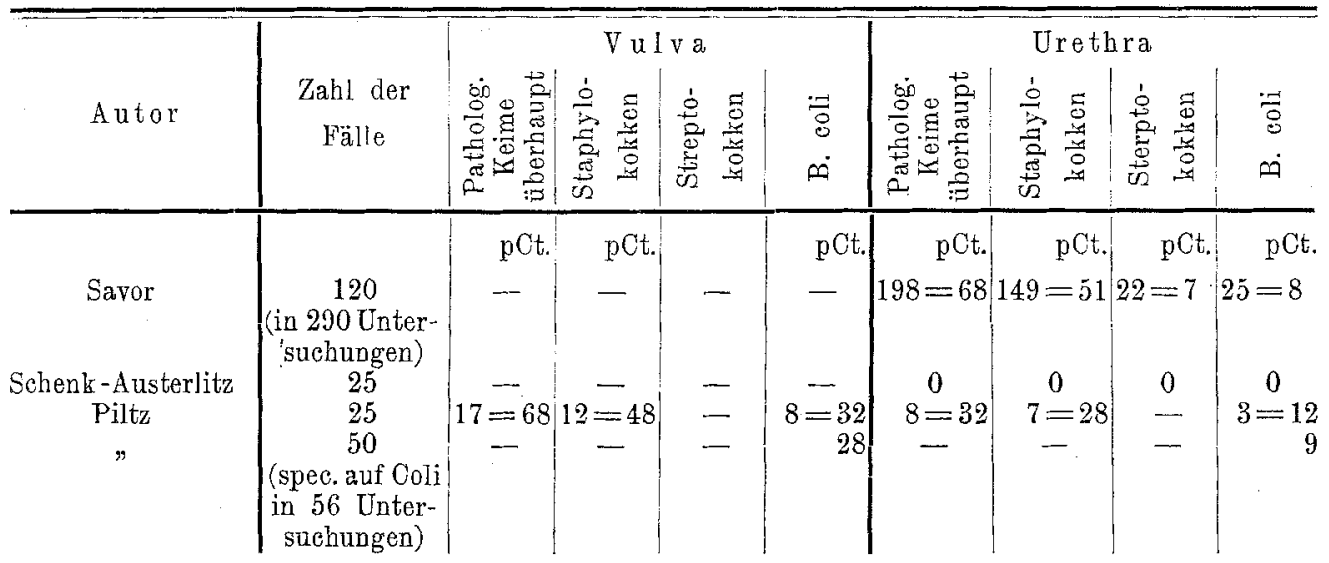

Tabelle III.

3. Wöchnerinnen.

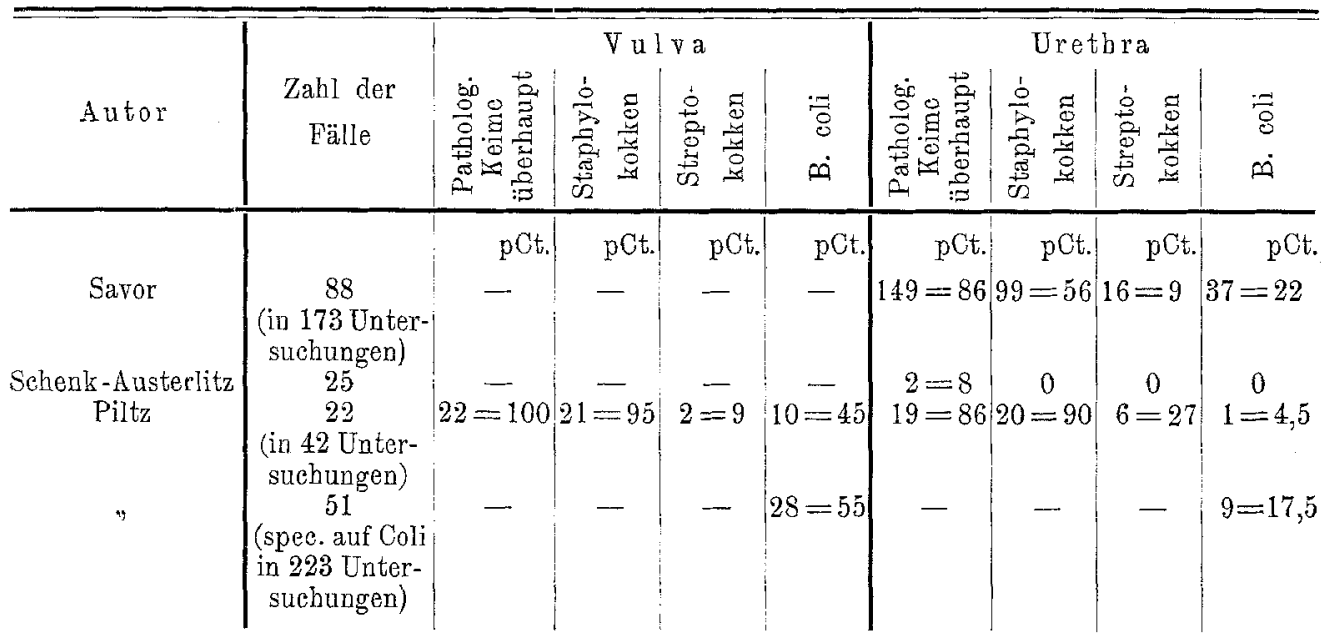

nisse einiger Untersuchungen skizzirt werden, um die bisher angenommene Frequenz des Bact. coli und das Hänfigkeitsverhältniss desselben gegenüber anderen pathogenen Keimen zu veransehaulichen. Wegen der unverkennbaren Abhängigkeit des Urethralkeimgehaltes von dem der Vulva (bezw. des Vestibulum) seien auch solche Befunde angeführt.

Die Tabellen lassen folgende allgemeine Schlüsse zu: Bei sehr vielen Frauen leben im Vulvarsecret pathogene Keime. In geringerer Frequenz kommen dieselben Keime in der Urethra vor. 
Am häufigsten findet man Staphylokokken - und hier dominirt der Staphylococcus albus über dem Staph. aureus -; es folgen mit geringerer Häufigkeit das Bact. coli und schliesslich in der kleinen Minderzahl der Streptococcus (und andere pathogene Keime).

Geht man mehr ins Detail, um aus der Gegenüberstellung einzelner Befunde tieferen Aufschluss zu gewinnen, so kommt man zu keinen rechten Vorstellungen. Nehmen wir nur die Frequenzziffer für das Bact. coli in der Urethra, so ist z. B. bei Nichtschwangeren von 0 pCt. (Schenk-Austerlitz) bis $66 \mathrm{pCt}$. (Baisch) ein zu grosser Sprung, als dass man ihn von vornherein mitmachen könnte. Baisch's Mitarbeiter Piltz fand hier nur 10 pCt. Bei Schwangeren und Wöchnerinnen ergeben sich die gleichen Divergenzen. Während Schenk und Austerlitz wieder negative Befunde erhielten, konnte Savor eine erhebliche Zunahme des Coligehaltes im Wochenbett feststellen. Die Resultato von Piltz schwanken an sich zu sehr, als dass sie sich zu bindenden Consequenzen eigneten. So traf er bei 22 Wöchnerinnen in 42 Untersuchungen das Bact. coli nur 1 mal in der Urethra an (= 4,5 pCt.), dagegen bei weiteren 51 Wöchnerinnen - jede durchschnittlich 4 mal untersucht -- in $17,6 \mathrm{pCt}$. Hieraus würde lediglich hervorgehen, dass mit der Häufigkeit der Untersuchungen die Höhe der Werthe entsprechend steigt. Schliesslich tritt auch ein auffallender Contrast zwischen den Beobachtungen Baisch's bei Bettlägrigen und denen der übrigen Autoren bei den doch gleichfalls bettlägrigen Wöchnerinnen deutlich zu Tage.

Da nun genauere Kenntnisse über den Coligehalt der weiblichen Urethra (und der Vulva) für die ganze Frage der Harninfection von grosser Bedeutung sind, stellten auch wir Untersuchungen an. Zugleich galt es, Stellung zu nehmen zu den neuen Werthen Baisch's, welche alle früheren Begriffe über den Haufen werfen und geeignet erscheinen, die urethrale Infectionsart mehr als je in den Vordergrund zu rücken.

Unsere Untersuchungsmethodik unterschied sich nur unwesentlich von der von Baisch und Piltz gewählten, indem wir aus gleicher Erwägung wie diese Autoren von einer vorherigen Desinfection absahen. Nach weiter Spreizung und sanftem Aufwärtsziehen der kleinen Labien wurde das Vestibularsecret mit ausgeglühter Platinnadel ausgestrichen. Nach erneutem Ausglühen ging die Nadel in die leicht zum Klaffen gebrachte Urethralöffnung ein und entnahm den Inhalt unter ausgiebigem Abstreifen der 
Urethralwände. Die Secrete wurden auf den Drigalski-Nährboden übertragen.

Auf diese Weise wurde das Bact. coli nachgewiesen:

bei 55 Schwangeren

im Vestibulum . . . $7 \mathrm{mal}=13$ pCt.
in der Urethra. . . $3 n=5,5 \%$

bei 103 Wöchnerinnen (durchschnittlich am 8. Tage untersucht)

im Vestibulum . . $44 \mathrm{mal}=42$ pCt.

in der Urethra . . $32 n=31$ "

bei 41 Wöchnerinnen (durchschnittlich am 4. Tage untersucht)

im Vestibulum . . $21 \mathrm{mal}=51 \mathrm{pCt}$.

in der Urethra. . $20 \%=50 \%$

bei 31 Wöchnerinnen (am 4. und später am 8. Tage untersucht)

Vestibulum:

Urethra:

4. Tag . $14 \mathrm{mal}=45 \mathrm{pCt} . \quad 14 \mathrm{mal}=45 \mathrm{pCt}$.

8. $" .10 n=32 n, 8 n=26 n$

bei 30 Aborten

$$
\begin{aligned}
& \text { im Vestibulum . . . } 12 \mathrm{mal}=40 \mathrm{pCt} . \\
& \text { in der Urethra . . } 13 \%=43 \mathrm{~m}
\end{aligned}
$$

Aus diesen Zahlen geht zur Evidenz hervor (mehr noch als bei Savor), dass im Wochenbett (und bei Aborten) eine erhebliche Frequenzsteigerung des Coligehaltes sowohl im Vestibulum als in der Urethra eintritt. Dabei zeigte sich, dass es nicht gleichgiltig ist, an welchem Wochenbetttage die Untersuchung vorgenommen wird. Während am 4. Tage ziemlich die Hälfte aller Wöehnerinnen das Bact. coli in der Urethra aufwiesen, war es am 8. Tag nur etwa im Drittel der Fälle zu finden. Zu einem ähnlichen Ergebnisse gelangte auch Piltz. Doch möchten wir diesen Unterschied mehr darauf zurückführen, dass unsere Wöchnerinnen durchschnittlich am 5. Tage das Bett verlassen. Bei Bettlägrigkeit (mit ihrer unvermeidlichen mechanischen Disposition) ist also die Frequenzziffer ungleich höher. Immerhin bleiben unsere Resultate hinter den Befunden Baisch's noch weit zurück. Der Grund hierfür liegt wohl in der Unzulänglichkeit aller einmaligen Untersuchungen. Gleichwohl wählten wir diese Methodik, um wenigstens relative Begriffe $z u$ erhalten und nicht in den Fehler früherer Forscher zu verfallen, verschieden häufige Untersuchungen bei einer begrenzten Zahl von Fällen zu einer einzigen Procentzahl zusammenzuwürfeln; wobei man sich nie des Eindrucks erwehren 
kann, dass noch mehr Untersuchungen auch noch höhere Werthe ergeben hätten.

Um solchen Bedenken aus dem Wege zu gehen und zugleich eine Ergänzung und Controle unserer eigenen Resultate zu gewinnen, wurde bei einer weiteren. Untersuchungsreihe das dem früheren entgegengesetzte Verfahren eingeschlagen: statt vieler Fälle mit einmaliger Untersuchung einzelne Fälle mit täglicher Secretcntnahme. Als Material glaubten wir zweckmässig Schwangere verwenden za sollen, da diese ja vorher die geringsten Werthe abgegeben batten. Von den täglich untersuchten 6 Hausschwangeren war das Bact. coli in früherer Untersuchung nur in einem Fall (No. 2) im Vestibulum, in keinem Falle in der Urethra nachgewiesen worden.

Tabelle IV.

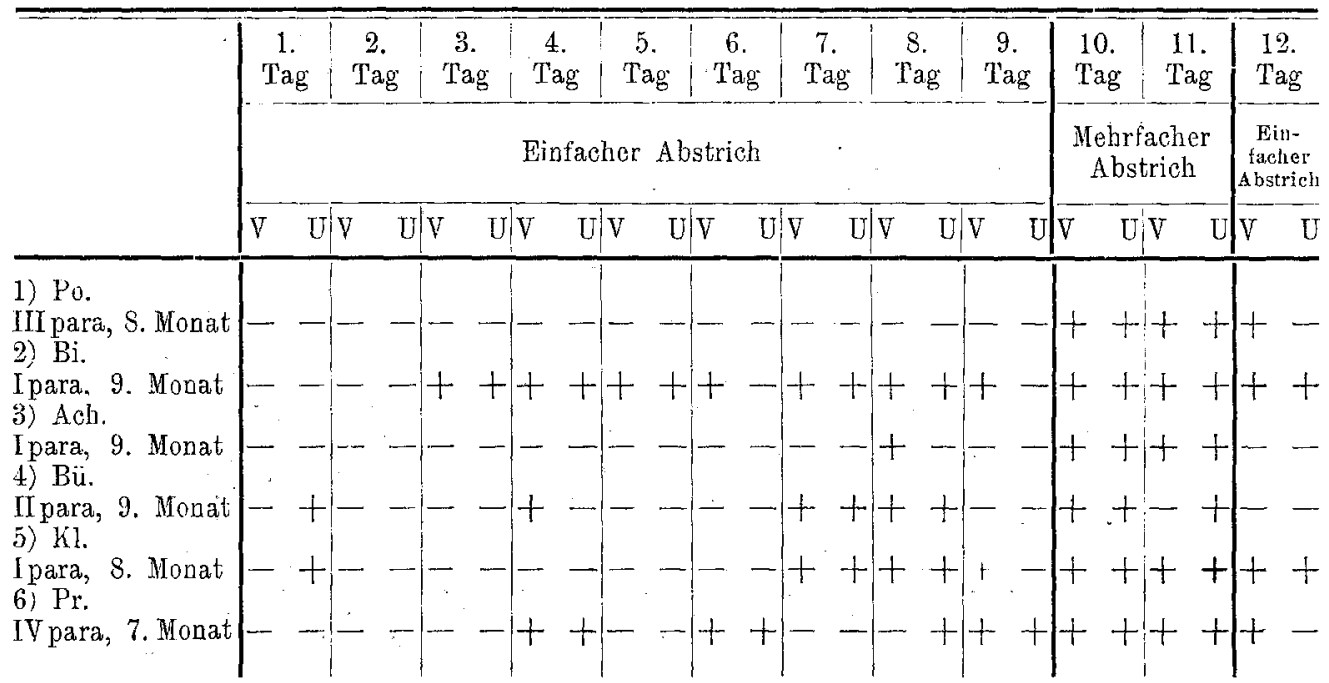

$\mathrm{V}=$ Vestibulum. $\mathrm{U}=$ Urethra.

Die Untersuchung (Tabelle 4) hatte das überraschende Ergebniss, dass bei allen 6 Schwangeren nach einer mehr weniger langen Zeit das Bact. coli im Vestibulum und in der Urethra angetroffen wurde. Schon am ersten Tage zeigte es sich - im Gegensatz zu dem früheren Befunde - bei 2 Schwangeren (in der Urethra, dagegen nicht im Vestibularsecret). Hieraus mag man schon ersehen, wie rom Zufall abhängig alle derartige Untersuchungen sind. Wir konnten uns sogar davon überzengen, dass eine einmalige, wenn auch gründliche, tägliche Secretentnahme 
nicht immer zum Ziele zu führen braucht. In Fall 1 and 3 waren erst dann Colibacillen in der Urethra nachzuweisen, als durch ein mehrfaches, aufeinanderfolgendes Ausstreichen jeder einzelne Winkel ausgekehrt war (10. und 11. Tag). Am 12. Tage, an welchem wieder ein einfacher Abstrich entnommen wurde, versagten beide Fälle abermals.

Aus dieser Untersuchung können wir wohl den weitgehenden Schluss ziehen, dass (allem Anschein nach) das Bact. coli ein regelmässiger Bewohner der weiblichen Urethra ist. Die Beweisführung gelingt nicht immer beim ersten Secretausstrich, sondern es bedarf mitunter, besonders wenn wenig Fluor vorhanden ist, einer längeren systematischen Untersuchung, um alle Furchen und Crypten, in welche sich das Bact. coli verkriecht, zu erschliessen. Dann aber gelingt es nach unserer Meinung immer, das Bact. nachzuweisen. Somit gehen wir noch einen Schritt weiter als Baisch, indem wir die Existenz des Bact. coli in jeder weiblichen Urethra - also auch ohne Bettlägrigkeit - annehmen. Unsere früheren Feststellungen (einmaliger Abstrich, einmalige Untersuchung) berechtigen aber wohl zu dem Schlusse, dass die gehäuften positiven Resultate im Wochenbett (und bei Aborten) mit einem zahlreichen Auftreten des Bact. coli zusammenhängen. Es scheint demnach der Wochenbettfluor begünstigend, der Schwangersehaftsfluor eher hinderlich auf die Vermehrung des Bact. coli einzuwirken. Für die Praxis ist diese Erkenntniss von grossem Werthe, da wohl jeder Katheterismus die Gefahr der Keimverschleppung in sich birgt, jedoch bei einer Wöchnerin mit ihrem stark keimhaltigen Urethralsecret mit ungleich höherer Wahrscheinlichkeit als bei einer Schwangeren.

Dass der Keimgehalt der Urethra im Wesentlichen von dem des Vestibulum abhängig ist, wie frühere Autoren betonten, geht auch aus unseren Befunden hervor. Desgleichen konnten wir uns von einem Parallelismus zwischen Urin- und Urethralkeimen überzeugen. Bei solchen Cystitiden, welche coliantagonistische Keime enthielten (z. B. Bac. Proteus), missglückte uns stets der Nachweis von Bact. coli in der Urethra, sei es, dass es fehlte oder durch Ueberwuchern der anderen Keime der Beachtung entging. Für solche (Ausnahme)-Fälle bedarf also unsere obige These der regelmässigen Ansässigkeit des Bact. coli in der weiblichen Urethra einer Einschränkung. Bei Cystitiden mit coli-indifferenten Keimen fand sich das Bact. coli schon bei einfacher einmaliger Untersuchung 
zumeist in der Urethra. Dieser Befund ist wichtig und rechtfertigend für die Annahme secundärer Coliinvasion in die Blase. Schlusssätze: Das Bact. coli ist ein (ziemlich) regelmässiger Bewohner der weiblichen Harnröhre. Der Schwangerschaftsfluor scheint sein Wachsthum zu hemmen; daher der seltene Nachweis bei gewöhnlicher Untersuchungsmethodik ( 5,5 pCt. der Schwangeren). Das Wochenbett begünstigt die Entwicklung und Vermehrung, wodurch die Thatsache erklärt wird, dass in der Hälfte der Wöchnerinnen schon bei einmaliger einfacher Untersuchung das Bact. coli sich in der Urethra nachweisen lässt.

\section{Der Infectionsmodus.}

Der Nachweis von Colibakterien in der Harnröhre harngesunder Erauen rechtfertigt von vornherein die Annahme einer exogenen (urethralen) Infection. Die Infection kann spontan durch selbstständiges Aufsteigen der Urethralkeime in die Blase oder durch mechanischen Import (Katheterismus) erfolgen. Letzterer Modus, schon 1870 von Olshausen erkannt und heute allgemein angenommen, wird namentlich durch die Thatsache gestützt, dass die Desinfection des Vestibulum und der Harnröhrenmündung ohne wesentliche Einwirkung auf die Bakterien des hinteren Harnröhrenabschnittes bleibt (Melchior, Piltz). Wir selbst können hierzu insofern einen Beitrag liefern, als bei einigen Wöchnerinnen, welche nachgewiesenermaassen Colibakterien in ihrer Urethra beherbergten und bei dem unter allen aseptischen Cautelen ausgeführten Katheterismus normalen keimfreien Urin darboten, am nächsten Tage eine ausgesprochene Blaseninfection (mit Bact. coli) festzustellen war. Allerdings steht man gegenwärtig hauptsächlich auf Grund zahlreicher Thierversuche, aber auch einzelner Beobachtungen am Krankenbette (Baisch) - cf. auch unsere Beobachtungen an Schwangeren (Casuist. Beitr. Fall 1-3) - mehr auf dem Standpunkte, dass ein einfacher Import von Keimen in die Blase nicht genügt, um eine Cystitis hervorzurufen; vielmehr wird die Mitwirkung gewisser Hilfsfactoren für erforderlich erachtet: Trauma, Congestion, Harnretention, Circulations- und Ernährungsstörungen (Thumim). Namentlich dem Trauma, einer häufigen Begleiterscheinung gynäkologischer Operationen, aber auch des Partus, kommt eine früher nicht beachtete Bedeutung zu, wie auch experi- 
mentell dargelegt wurde (Dubolt1), Baisch). Ueber die Bewerthung der Harnretention besteht noch keine Congruenz der Meinungen. Schon Rovsing (1898) machte gegen die einseitige Ueberschätzung derselben Front. Baisch gelangte auf experimentellem Wege (Versuche an Hündinnen) zu gleicher Anschauung. Wohl aber scheint die partielle Harnretention, die continuirliche unvollständige Urinentleerung, durch die für Bakterien günstigen Entwickelungsbedingungen zur Infection zu disponiren. Im Allgemeinen wird man sagen müssen, dass eine Gesamtwirkung der verschiedensten Hilfskräfte bei der Entstehung der Cystitis im Spiele ist, wie sie in der Schwangerschaft, im Wochenbett, bei entzündlichen Veränderungen der Umgebung, beim Trauma, bei Prolapsen, bei Blasenstörungen in Folge Rückenmarksläsion etc. deutlich zu Tage tritt.

Schliesslich ist als Cystitisprovocation par excellence der Dauerkatheterismus zu nennen. Die rcizende und eystitisfördernde Wirkung desselben ist längst bekannt. Jedoch findet sich in der ganzen Literatur - soweit sie uns vorliegt - nirgends Erwähnung gethan, dass der Dauerkatheter speciell der Coliinfection Vorschub leistet2). Andere Cystitiden scheinen uns hierbei zu den Seltenheiten zu gehören. Wenn nicht therapeutisch eingegriffen wurde, verursachte der Pezzer-Katheter in allen Fällen eine Colicystitis, die gewöhnlich nach 24 Stunden die ausgesprochensten Symptome zeigte und auch nach Entfernung des Katheters bestehen blieb. Selbst dann, wenn schon vor Einführung eine (anderskeimige) Cystitis bestand, sahen wir das Bact. coli immer hinzutreten, welches dann mitunter wieder verschwand, zumeist aber dauernd blieb und dann häufig die anderen Bakterien gänzlich ablöste.

Wenn im bisher Gesagten unsere Meinung dahin zum Ausdruck gebracht wurde, dass die überwiegende Mehrzahl der Coli-Harninfectionen von den Urethralkeimen ihren Ausgang nimmt, somit also die Blase wiederum den Ausgangspunkt für die Infection der oberen Harnwege vorstellt, so bleibt doch ein kleinerer Theil derselben dem endogenen Modus, der Descension, zugedacht, sei es, dass das Bact. coli auf dem Umwege über das Blut oder auf

1) Cit. nach Baisch.

2) Unsere Erfahrungen beziehen sich in erster Linie auf den Pezzer'schen Gummikatheter; die Untersuchungen mit dem Skene'schen Glaskatheter sind noch nicht abgeschlossen. 
directem Wege vom Darm in die Harnorgane überwandert. Diese endogene Theorie, zuerst von der Guyon'schen Schule aufgestellt und verfochten und in erster Linie für die Infection der oberen Harnwege (Pyelitis) in Anspruch genommen, hat namentlich in den letzten Jahren vielfach energischen Widerspruch erfahren (0 pitz a.a.). Eine strikte Beweisführung für oder gegen dieselbe - sie ist auch heute noch eine blosse Theorie - erscheint aber vor der Hand unmöglich. Schon die Frage der Bakteriendurchlässigkeit der normalen Darmschleimhaut und Niere ist zur Zeit noch unentschieden. Auf der einen Seite in Abrede gestellt (Neisser, Opitz, Wyssokowicz a. a.), wird sie andererseitc bejaht (Holle, Streng, Pawlowsky, Rolly u. a.). Noch verwickelter wird die Frage, wenn diese Organe geschädigt sind, ein Vorkommnis, das in der Schwangerschaft und im Wochenbett relativ häufig anzutreffen ist. In seiner Begründung führt Opitz die von Pfeiffer und Kolle gemachte Beobachtung an, dass im Blute normaler Menschen keine Coli-Antikörper kreisen, ferner die Sterilität des Bruehwassers. Während aber erstere Thatsache sich eben auf normale Verhältnisse bezieht, ist bei letzterem Befund zu wenig die baktericide Eigenschaft des Bruchwassers berücksichtigt. In schwereren Fällen konnte Brentano denn anch das Bact. coli aus dem Bruchwasser züchten. Auch der seltene Nachweis von Colibakterien im Blute ist nicht ausschlaggebend, da er die directe Ueberwanderung nicht betrifft. Dieser letztere Modus wird aber gerade in neuester Zeit wieder ins Feld geführt (Mirabeau). Weiter begegnete man den "Troubles gastro-intestinaux" der französischen Schule mit dem Einwande, dass dieselben keine Vorboten, sondern eine Begleiterscheinung der beginnenden oder vorhandenen Urininfection wären. Den Kinderärzten, die der Darminfection ein gewisses Recht einräumen (Escherich, Trumpp, Finkelstein u. a.) wurde vorgehalten, dass die Coliinfectionen weit häufiger bei Mädehen als bei Knaben aufträten.

Auch an den Thierexperimenten, welche zugunsten der endogenen Theorie sprachen, wurde scharfe Kritik geübt. Gegen die bekannten Versuche von Posner und Lewin (Coli-Harninfectionen nach Harnröhrenunterbindung ond Rectumversehluss bei Kaninchen) nahm Marcus Stellung, indem er die Versuchsanordnung für nicht einwandfrei erklärte. Gegen die Experimente Wreden's (ColiHarninfection nach Verletzung der Mastdarmschleimhaut in der Höhe der Prostata) protestirte Faltin. 
Aus eigenen Versuchen an Kaninchen gelangten wir zu der Vorstellung, dass die alleinige Rectumunterbindung genügt, um nach einigen Tagen die Colibakterien in die Blutbahn eintreten zu lassen. Ihr cultureller Nachweis in dem Milz- und Nierengewebe war dann leicht. Schnittpräparate der Milz liessen in einem Falle typische gramentfärbte Kurzstäbchen erkennen. Aus dem Urin waren sie jedoch nicht züchtbar. Ebenso waren Blasen- oder Nierenveränderungen nicht eingetreten. Eine chronische Obstipation konnten wir trotz grosser Opium- und Atropingaben bei Kaninchen nicht erzielen. Wird man nun allen derartigen Thierversuchen (Rectumunterbindung etc.) immer den Vorhalt machen dürfen, dass sie gewaltsam sind und nicht willkürlich auf die menschlichen Verbältnisse übertragbar, so haben doch zahlreiche Versuche beweisend dargethan, dass wenigstens beim Thier unter gewissen Voraussetzungen die Darmschleimhaut den Durchtritt von Bakterien nicht zu hindern vermag.

Gehen wir auf die am Menschen gemachten klinischen Erfahrungen über, so erseheint uns namentlich eine Beobachtung Trumpp's bemerkenswerth. Bei gelegentlich einer Hausepidemie mit Enteritis follicularis angestellten systematischen Untersuchungen fand Trumpp unter 17 Kindern 14 mal das Bact. coli im Urin: und zwar 5 mal bei Knaben. Ein derartiger Befund darf wohl nicht als zufällig angesehen werden. In gleicher Weise wie beim Kinde müssen auch beim Erwachsenen vorausgehende Darmstörungen, wie sie z. B. Opitz im Falle 13, 48, 57, 64 citirt, in den Bereich der endogenen Infectionsmöglichkeit gezogen werden. Wir selbst sahen eine Coli-Harninfection bei einer Wöchnerin, welche an einer 8 tägigen Obstipation litt und auf geeignete Therapie hin eine 7 malige reichliche übelriechende Entleerung hatte. Der definitive Beweis steht sowohl für diesen wie für alle ähnlichen Fälle der Literatur aus. Daher wird der Gegner der endogenen Infection auch schwer von der Richtigkeit dieser "Theorie" zu überzeugen sein. Andrerseits sind aber auch die Anhänger der Theorie in der Classificirung ihrer Fälle oft genug einseitig gewesen. So scheint uns von den 35 Fällen'Suter's von endogener Coliinfection kein einziger stichkräftig zu sein; ferner scheint uns Mirabeau die unleugbaren innigen Beziehungen der rechten Niere zum Darm für den Bakterienübertritt $\mathrm{zu}$ hoch anzuschlagen.

Schlusssätze: Die Infection der Harnwege mit Bact. coli erfolgt zumeist durch (spontane oder mechanische) Ascendenz von 
der Urethra (bezw. Blase) her. Für einzelne Fälle ist der endogene Modus (Descendenz) nicht von der Hand zu weisen. Der Vorgang der Infection ist dabei an eine gleichzeitige Mitwirkung von Hülfsfactoren (Complicationen) gebunden. Unter letzteren nimmt der Dauerkatheterismus (Pezzer) die erste Stelle ein, weil er immer eine Colicystitis im Gefolge hat.

\section{Die Pathogenität des Bact. coli.}

Das Punctum saliens der grossen Colidebatte bildet naturgemäss die Pathogenitätsfrage; ihr gegenüber hat der Meinungsstreit über den Infectionsmodus nur untergeordnete Bedeutung. Nachdem man in vielen Beobachtungen der letzten Jahre das Bact. coli als selbstständigen Krankheitserreger kennen gelernt ["Colibacillosen" Gilbert")] und in Abscessen und Entzündungsherden der verschiedensten Organe, bei Wundinfection, Peritonitis, puerperaler Sepsis, Tympania uteri, Cholecystitis, Augenblennorrhoe und schliesslich in der Mehrzahl der Harninfectionen nachgewiesen hat, müsste jeglicher Zweifel an der pathogenen Bedeutung dieses Bacterium gehoben sein. In Wirklichkeit aber finden wir auch hier wieder grosse Unklarheit und Verschiedenheit der Meinungen. Immer wieder wird der Einwand gemacht, dass das Bact. coli vielleieht nur ein zufälliger, den wirklichen Entzündungserregern beigesellter Parasit sei, der vermöge seiner grossen Vermehrungsfähigkeit in der Quantität leicht die anderen Keime überflügele und durch sein elektives, üppiges Wachsthum auf den Nährböden die Anffindung der ursprünglichen Erreger sehr erschwere oder sogar vereitle. In der Betonung der Harmlosigkeit des Bact. coli für die Blase geht Rovsing soweit, aus therapeutischen Grïnden zur Bekämpfung und Verdrängung anderer Cystitiskeime an eine künstliche Infection der Blase mit Colikeimen zu denken, ein Vorsehlag, den - beiläufig bemerkt - Faltin gerade umkehren möchte.

Die Beurtheilung der Bedeutung des Bact. coli für die Blase ist um so schwieriger, als die in der Entstehung begriffene Cystitis nur selten zur Beobachtung kommt. Wenn erst subjective Beschwerden auftreten, dann ist die Cystitis meist eine vollendete Thatsache und die Entscheidung, ob das Bact. col primär oder secundär betheiligt ist, häufig nur muthmasslich zu treffen. Dieser Schwierigkeit waren wir enthoben, als wir in dem Dauer-

1) Cit, nach Escherich-Pfaundler. 
katheterismus eine specifische Provocation der Colicystitis erkannt hatten. Durch beliebig häufige Entnahme und Untersuchung des Urins konnte nunmehr das erste Auftreten der Colistäbchen und das erste Entstehen von Cystitissymptomen (Eiterzellen) verfolgt werden. Die Entnahme geschah in der Weise, dass zuerst eine grössere Menge Harnes abgelassen wurde, um den distal von der Klemme gelegenen Schlauchabschnitt auszuspülen und hierdurch vor einer unwillkommenen Beimischung gänzlich fernstehender Keime nach Möglichkeit zu schützen. Der Rest wurde in sterilen Reagenzgläsern aufgefangen; die Sedimentirung erfolgte in sterilen Spitzgläsern durch Centrifuge.

Aus unseren vielfachen Erfahrungen seien nur einzelne Fälle hier aufgeführt; in den casuistischen Beiträgen finden sich dann noch weitere Beispiele.

I. Frl. A. Laparotomie. Dauerkatheter. 22. 9. 1 Uhr Nachm.

1. Urin: am 8. 9. untersucbt: Einzelne Leukocyten und Epithel. Sonst o. B. - Culturell: negativ.

2. Urin: 22. 9. 4 Uhr Nachm. (3 Stunden nach Einführung des Pezzer-Katheters) dunkelgelb, klar, sauer. - Sediment: Einzelne Epithelien und Lenkocyten. Keine Bakterien. - Gramfärbung: Keine Bakterien. - Boullon, Agar. Drigalski-Agar: nach 24 und 48 Std. steril.

3. Urin: 23. 9. 12 Uhr dunkelgelb, leicht unklar, sauer. - Sediment: Vereinzelte Leukocyten. Gewimmel von mässig beweglichen Stäbchen. - Gramfärbung: Massenhaft gramnegative Kurzstäbchen in Reininfection. - Bouillon, Agar, Glycerinagar, Drigalskiagar: Reinculturen vou B. coli. - Gelatine: nicht verflüssigt.

4. Urin: 24. 9. $9 \mathrm{Uhr}$ Vorm.: dunkelgelb, milchig trüb, saner. Sediment: Sehr zahlreiche Leukocyten und massenhaft gramnegative Stäbchen, zum Theil in Ketten aneinanderliegend (Reininfection). Bouillon, Agar, Glycerinagar, Drigalskiagar, Gelatine: B. coli in Reincultur.

II. Frau P. 2. 7. Vor 8 Tagen Partus. Seitdem Urinverhaltung. Ischuria paradoxa. Harnblase bis Nabelhöhe gefüllt. Somnolenz. Katheterismus: 3 Liter Urin; alkalisch. - Sediment: Viele Erythrocyten, einzelne Leukocyten. Viele Epithelien. Keine Bakterien. - Bouillon, Drigalskiagar: steril nach 24 Stunden. Nach 10 Minuten Einführung des Dauerkatheters. Hierbei wieder $3 / 4$ Liter Urin (Ureteren- und Nierenbeckenurin); schwach sauer. - Sediment: Sehr viele Erythrocyten. Sehr viele hyaline Cylinder. Sonst o. B. Keine Bakterien. - Bouillon, Drigalskiagar: steril nach 24 Stunden.

3. 7. Urin: sauer; trüb. - Sediment: Sehr zahlreiche Erythrocyten und sehr viele (polynueleäre) Leukocyten. Viele hyaline Cylinder. Gewimmel von gramnegativen Kurzstäbchen. - Culturell: B. coli in Reincultur.

4. 7. Urin: sauer, sehr trüb. -- Sediment: Nur noch vereinzelte Erythrocyten. Massenhaft Eiterzellen und Gewimmel von gramnegativen Stäbchen. - Culturell: B. coli (rein). 
III. Frau D. Eklampsie. Operative Entbindung. 21. 8.:41/2 Uhr Nachm. Einführung des Dauerkatheters.

1) 21. 8. $4^{1 / 2}$ Uhr Nachm. Urin: sauer, dunkelbraun. - Sediment: Massenhaft Erythrocyten. Viele lyyaline und granulirte Cylinder. Keine Bakterien. - Trockenausstrich: Massenhafte Erythrocyten und entsprechende Menge weisser Blutzellen. Keine Bakterien. - Sedimentausstrich auf Bouillon, Agar, Drigalskiagar: steril nach 24 und 48 Std.

2) 21. 8. 111/2 Uhr Nachm. Urin: sauer, gelbbraun. - Sediment: Zahlreiche Erythrocyten, einzelne Leukocyten. Viele Cylinder, einzelne Epithelien. Vereinzelte Stäbchen. - Trockenausstrich: Gleicher Blutzellenbefund. Vereinzelte plumpe Stäbchen, sonst keine Bakterien. - Gramfärbung: Mehrere gramnegative Stäbclsen. - Sedimentausstrich auf Bouillon, Glycerinagar, Drigalskiagar: Reincultur von B. coli.

3) 22. 8. $1 \frac{1 / 2}{2}$ Uhr Vorm. Urin: gelbbraun, stark sauer. - Sediment: wie vorher, nur zahlreichere Stäbchen. - Trockenpräparat nach Gram: Gramnegative Stäbchen, sonst keine Bakterien. - Bouillon. Agar, Glycerinagar: Reinculturen von B. coli. (Bouillon: starke Indolreaction nach 12 Stunden.)

4) 22. 8. 8 Uhr Vorm. Urin: dunkelgelb, leicht getrübt, stark sauer, fader Geruch. - Sediment: Zahlreiche Leukocyten, ganz vereinzelte Erythrocyten. Massenhaft gramnegative Stäbchen (Reininfection). - Culturell: B. coli (rein).

5) 22. 8. 10 Ubr Vorm. Urin: stark sauer, leicht getrübt, fader Geruch. - Sediment: Grosse Mengen Leukocyten, zum Theil zu Haufen geballt. Ganz vereinzelte Erythrocyten. Einzelne Cylinder. Gewimmel von Stäbchen. - Gramfärbung: Massenhaft gramnegative Kurzstäbchen (Monoinfection). - Culturell: B. coli (rein).

6) 22. 8. 4 Uhr Nachm. Urin: stark sauer, opalescirende Trübung (starker Bodensatz). - Sediment: Massenhaft Eiterzellen und gramnegative Stäbchen, zum Theil in Haufen zusammenliegend (Reininfection). - Culturell: B. coli (rein).

IV. Frl. P. Eklampsie. Operative Entbindung. 11. 8. 8 Uhr Vorm. Einführung des Pezzer-Katheters.

1) 11. 8. 8 Uhr Vorm. Urin: hell, klar, stark sauer. Alb. + (7 pCt.). - Sediment: Sehr viele Cylinder. Einzehne Leukocyten. Sonst o. B. Keine Bakterien. - Trockenpräparat: Keine Bakterien. - Bonillon, Agar, Hydrocelenagar, Drigalskiagar: steril nach 24 und 48 Stunden.

2) 11. 8. $11 \frac{1}{2}$ Uhr Yorm. Urin: hell, klar, stark suuer. - Sediment: Cylinder; einzelne Leukocyten sonst 0. B. - Trockenpräparat: o. B. - Agar, Hydrocelenagar, Drigalskiagar: steril nach 24 u. 48 Std. - Bouillon: trüb nach 24 Stà. (Staphylokokken). Verunreinigung!1).

3) 11. 8. 2 Uhr Nachm. Urin: hell, klar, stark sauer. - Sediment: Viele Cylinder, Leukocyten in etwas grösserer Menge als vorher, mitunter zu mehreren zusammẹnliegend. Keine Bakterien. - Trockenpräparat: Leukocyten, sonst o. B. - Bouilion, Agar, Hydrocelenagar, Drigalskiagar: steril nach 24 und 48 Stunder.

4) $11.8 .4 \frac{1}{2}$ Uhr Nachm. Urin: hell, klar, stark sauer. - Sediment: Weniger viele Cylinder. Leukocyten gegen 3) etwas vermehrt. Sonst o. B. Keine Bakterien. - Trockenpräparat: Leukocyten, sonst o. B. - Bouillon, Agar, Hydrocelenagar, Drigalskiagar: steril nach 24 und 48 Stunden.

1) Urin später wieder steril. 
5) 11. 8. 7 Uhr Nachm. Urin: hell, klar, stark sauer. - Sediment: wie unter 4). - Gleiche Näbrböden: steril nach 24 und 48 Stunden.

6) 11. 8. 9 Uhr Nachm. Lrin: hell, klar, sauer. - Ueberimpfung auf Drigalskiagar: steril nach 24 Stunden. (Sonst keine Untersuchung.)

7) 12. 8. 1 Uhr Vorm. Urin: hell, klar, stark sauer. Alb. +; Sediment: nur verimpft. - Bouillon: trüb nach 12 Stunden. -- Trockenpräparat: gramnegative Stäbchen und grampositive Staphylokokken. -Aussaat auf Drigalskiagar: B. coli in Reincultur. - Hydrocelenagar: einzelne Colonien nach 24 Stunden gewachsen. - Trockenpräparat: gramnegative Stäbchen und Staphylokokken. - Aussaat auf Drigalskiagar: B. coli in Reincultur. - Drigalskiagar: nach 12 Stunden einzelne Colonien von B. coli in Reincultur gewachsen.

8) 12. 8. $8 \mathrm{Ubr}$ Vorm. Urin: hell, klar, stark sauer. Alb. + (1 p.M.). - Sediment: Viele Cylinder. Sehr viele Leukocyten, zum Theil zu grösseren und kleineren Haufen zusammengeballt. Viele Bakterienhaufen (aus Stäbchen bestehend). Viele kurze, plumpe Einzelstäbchen. - Trockenpräparat: Viele Eiterzellen. Viele gramnegative Stäbchen, zum Theil auch in Kettenform. Vereinzelte (grampositive) Kokken. Bouillon: trüb, B. coli in Reincultur. - Agar: über 20 Colonien gewachsen. B. coli (dazwischen ganz vereinzelte Kokken). - Drigalskiagar: B. coli in Massen gewachsen (Reincultur). - Hydrocelenagar: 6 Colonien. B. coli, ferner einzelne Kokken.

9) 12. 8. 11 Uhr Vorm. Urin: hellgelb, leicht opalescirende Trübung; stark sauer. - Sediment: wie unter 8). - Bouillon: stark getrübt. B. coli in Reincultur. - Agar: Reichliches Wachsthum. B. coli in Reincultur. - Drigalskiagar: B. coli in Massen gewachsen. (Reincultur.)

10) 12. 8. 1 Uhr Nachm. (Dauerkatheter entfernt.) Urin: bell, opalescirende Trübung; stark sauer. - Sediment: Viele Cylinder. Sehr zahIreiche Leukocyten, zum Theil in grösseren und kleineren Haufen. Massenhaft gramnegative Stäbchen und vereinzelte Kokken. - Bouillon, Agar, Drigalskiagar: Reincultur von B. coli. - Hydrocelenagar: massenhaft Colistäbchen, vereinzelte grampositive Kokken, - Aussaat auf Drigalskiagar: B. coli (rein).

11) 12. 8. 7 Uhr Nachm. (Einfacher Katheterismus.) Urin: hell, stärkere Trübung; stark sauer. - Sediment: wie unter 10).-Culturell: hauptsächlich B. coli. Ferner vereinzelte grampositive Diplokokken.

12) 13. 8. 8 Uhr Vorm. (Katheterismus.) Urin: gelb, stark getrübt stark sauer. - Sediment: sehr zahlreiche Eiterzellen und massenhaft, gramnegative Stäbchen (Monoinfection). - Culturell: B. coli (rein).

18) 13. 8. 5 Uhr Nachm. (Katheterismus.) Urin und Sediment: wie unter 12). - Culturell: B. coli in Reincultur.

Während Fall 1-3 Beispiele reiner Colicystitis vorstellen, gebört Fall 4 zur Kategorie jener Cystitiden (nach Dauerkatheterismus), in welchen das Bact. coli bei seinem ersten Auftreten mit einem anderen Keime vergesellschaftet ist. Wir selbst sind auf Grund unserer zahlreichen Beobachtungen geneigt, auch hier das Bact. coli als den ursprünglichen Erreger der Cystitis aufzufassen und nicht von einer Mischinfection, als vielmehr von einer zufälligen, 
belanglosen Beimischung zu sprechen. Der nicht steril blcibende Katheter bietet zu Verunreinigungen genügend Gelegenbeit.

In der Annahme und Behauptung einer primären Colicystitis stehen wir nicht allein da. Schon Goldberg, Faltin, Liebermeister u. A. konnten Mono-Coliinfectionen von Anfang an verfolgen. Auch wird in den meisten neueren Arbeiten und Handbüchern des Bact. coli als selbstständigen Cystitiserregers gedacht. Jedoch scheinen unsere Beobachtungen exacter zu sein als alle früheren, da wir die Urine schon vor der — theilweise künstlich durch Dauerkatheter gesetzten - Erkrankung untersuchen und die ersten cystitischen Veränderungen genau controliren konnten. Derartige exacte Beobachtungen sind aber nothwendig, um die primäre Schädlichkeit des Bact. coli für die Blase endgültig festzunageln.

Somit treten wir in Widerspruch einerseits zu Rovsing, andererseits zu Baisch. Den Befund einer secundären Coliinvasion als ein häufiges Vorkommniss anerkennend, müssen wir die von Baisch gefolgerte Verallgemeinerung dieses Vorganges auf die gesammten Colicystitiden ablehnen.

Die Beweisführung einer primären Colicystitis war voranszuschicken, um auf diesem strittigen Felde unsere Stellungnahme zu präcisiren und zugleich eine Basis zu gewinnen, auf welcher der Beurtheilung der Pathogenität des Bact. coli an sich nähergetreten werden kann. Die Thatsache, dass das Bact. coli befähigt ist, selbstständig eine Cystitis zu erzeugen oder eine schon vorhandene anderskeimige Cystitis fortzuführen, genügt njcht, um den Grad der Pathogenität für die Harnwege zu kennzeichnen. Einerseits braucht ein einfacher Import von Colikeimen keine Entzündung zur Folge zu haben - wie dies experimentell auch für andere Hohlorgane (Nierenbecken, Gallenblase) nachgewiesen wurde - andererseits ist bekannt, dass bei Pyelitiszuständen, bei Darmperforationen in die Blase ete. letztere continuirlich mit Colibakterien überschwemmt werden kann, ohne dass die Blasenschleimhaut in Mitleidenschaft gezogen wird.

Von der Erwägung ausgehend, dass ein zu Milliarden im menschlichen Organismus (Darm) lebendes Bacterium an sich nicht pathogen sein könne, hat man schon längst an Virulenzunterschiede gedacht und ist in diesem Gedanken bestärkt worden durch die Beobachtungen von Lesage und Macaign $\mathrm{e}^{1}$ ), nach welchen Autoren das Bact.

1) Cit. nach Escherieh-Pfaundler. 
coli von darmkranken Individuen einen deutlichen Energiezuwachs besitzen soll. Diese Virulenzsteigerung, heute ziemlich allgemein anerkannt, ist für viele Fälle sicherlich ätiogisch wichtig, kommt aber wohl für das Gros der Coliharninfectionen, wie wir sie z. B. beobachteten, kaum in Betracht. In neuester Zeit. hat Schmidt versucht, vermittels der Hämolyse Virulenzunterschiede aufzudecken, ohne jedoch Erfolge aufweisen zu können. Wir versuchten gleichfalls dem Problem näher zu rücken, indem wir von dem Gesichtspunkte der Pyogenität aus, der Eiterbildung, die.Lösung erhofften. In der Voraussetzung, dass die Colibacillen bei Bakteriurie nicht pyogen, dagegen bei Cystitis und Pyelitis pyogen, also erstere wenig oder gar nicht pathogen, letztere sensu strictiori pathogen wären, wurden die Urine direct in Dosen von $0,2-0,3 \mathrm{ccm}$ weissen Mäusen subcutan einverleibt. Die eiterhaltigen Urine wurden erst injicirt, nachdem sich der Eiter am Boden des Reagenzglases abgesetzt hatte. Die Versuche ergaben ein vollständig negatives Resultat. Enthielt der Urin, gleichgültig, ob er einer Bakteriurie oder einer Cystitis entstammte, viele Bakterien, so starben die Thiere nach kürzerer oder längerer Zeit (12 Stunden bis 4 Tage) an allgemeiner Colisepsis (Bact. coli im Herzblut ${ }^{1}$ ); bei geringem Bakteriengehalt blieben sie am Leben und zeigten keine Störungen. Eiterbildung (Abscesse, eitrige Peritonitis) wurde nie beobachtet. Nach dem gegenwärtigen Stande der Forschung werden wir uns daher mit der Annahme bescheiden müssen, dass es zwar virulenzgesteigerte Colibakterien (bei Darmstörungen) giebt, dass aber für gewöhnlich die veränderten Lebensbedingungen hinreichen, um aus einem Saprophyten einen pathogenen Keim entstehen zu lassen. Die Fähigkeit der Eiterbildung scheint dabei an gewisse Störungen von Seiten der Harnorgane (agents provocateurs) geknüpft zu sein.

Schon hieraus ist ersichtlich, dass das Bact. coli hinter anderen Cystitiskeimen - z. B. den Gonokokken - oder den harnstoffzersetzenden Bakterien, welche allein durch die Fähigkeit der Ammoniakbildung sich die Blasenschleimhaut in breitestem Maasse zugänglich machen - an pathogener Einwirkung erbeblich zurücktritt. Dafür sprechen auch die geringfügigen Veränderungen der Blasenschlejmhaut bei Colicystitis, welche zumeist nur in einer wenig ausgeprägten Injection der Trigonumgefässe bestehen. Rov-

1) Die Controllthiere, mit der gleichen Dosis eben derselben abgekochten und filtrirten Urine geimpft, blieben munter. 
sing glaubt den Nachweis einer relativ geringen Schädlichkeit auch für die oberen Harnwege führen zu können. Bei Operationen fand er im Gegensatz zu den tiefgreifenden Schädigungen harnstoffzersetzender Mikroben die Schleimhant des Nierenbeckens blass und normal. Während er bei Coliinfection die Niere spalten, den Eiter über das Nierenparenchym ausströmen lassen und dasselbe darauf ohne nennenswerthe Reaction wieder complet verschliessen konnte, wäre dies nach seiner Ansicht bei Entzündungszuständen mit harnstoffzersetzenden Keimen ein schlimmer Fehler gewesen. Die Rovsing'schen Beobachtungen überzeugen um so mehr, als auch bei Obductionen mildere Veränderungen angetroffen wurden. Baisch konnte in einem Falle die Colibakterien nur im Nierenbecken, in den Harnkanälchen und in den nekrotischen Partien und Abscessen nachweisen, während Staphylokokken in das Nierengewebe selbst eingedrungen waren. v. Wunschheim kommt zu dem Schlusse, dass die durch Bact. coli verursachten Pyelonephritiden sich von den durch Staphylo- und Streptokokken entstandenen, nicht bloss durch das Fehlen der mit letateren Bakterien meist verbundenen Pyämie, sondern auch mikroskopiseh durch den geringeren Zerfail des Gewebes etc. unterscheiden. Die von Schmidt-Asch off beschriebene Nekrose des Nierengewebes traf er nicht so häufig an.

Nach alledem ist an einer weniger tief eingreifenden Schädlichkeit von Seiten des Bact. coli nicht zu zweifeln. Man wird aber bei der Beurtheilung der Dignität des Bact. coli zwei Factoren mehr als früher berücksichtigen müssen: die enorme Vermehrungsfähigkeit und die Eigenbeweglichkeit. Wir konnten uns selbst in einem Falle davon überzeugen, dass 5 Tage nach eingetretener Colicystitis (in Folge Dauerkatheterismus) in beiden Ureteren Colikeime nachweisbar waren. Die Infection springt also leicht auf die oberen Harnwege äber. Thatsächlich verdankt denn auch der grösste Theil aller Pyelitiden (in der Schwangerschaft und im Wochenbett) diesem Keime seine Entstehung. Das Bact. coli wiegt also seine geringere pathogene Bedeutung durch die Häufigkeit dieser Affectionen gewissermaassen wieder auf. Ferner wird man die aeut verlaufenden Fälle (mit ihren geringfügigeren pathologischen Veränderungen) von den chronischen Formen trennen müssen, welche letztere doch nicht allzu selten das Nierengewebe selbst angreifen und in der Rindensebicht zahlreiche miliare Abscesse bilden oder aber in einen einzigen Nierenabscess übergehen und dann das Nierengewebe zum Schwunde bringen. Rovsing konnte in einem 
Falle sogar Perforation eines Abscesses in die Bauchhöhle beobachten. Schliesslich ist noch der ausgesprochenen Toxinproduction Erwähnong zu thun, welche namentlich dem Krankheitsbilde der acuten Pyelitis den Ausdruck einer schweren, septikämischen Erkrankung verleiht.

Schlusssätze: Das Bact. coli., einmal in die Harnwege eingedrungen, ist ein beharrlicher, keineswegs harmloser Parasit, da es vermöge seiner Eigenschaften der starken Vermehrungsfähigkeit und Eigenbewegung sich den ganzen Harntract erobern kann und und bei Eintritt irgend welcher Complicationen (also namentlich in der Schwangerschaft und im Wochenbett) befähigt ist, primär Entzündungszustände auszulösen (Cystitis, Pyelitis).

Das Bact. coli ist demnach ein echter Cystitiserreger, welcher durch diese Eigenschaft auch in Stand gesetzt wird, anderskeimige Cystitiden - nach Verdrängung der ursprünglichen Erreger fortzuführen.

Die Schädigungen scheinen hinter den Einwirkungen anderer pathogener Bakterien weit zurückzutreten, können aber bei der chronischen Form der Pyelitis gleiche Intensität erreichen.

Durch das schnelle Vordringen in die Ureteren ist das Bact. coli die häufigste Ursache der Pyelitiden (in der Schwangerschaft und im Wochenbett).

Das Bact. coli. wirkt schliesslich - namentlich bei Pyelitis acuta - durch eine starke Toxinproduction schädigend auf den Organismus ein.

\section{Die Harninfectionen.}

Allgemeiner Theil.

Das Vorgehen einzelner Autoren, bei einer Allgemeinbetrachtung der Harninfectionen statt der Krankheitsbegriffe (Cystitis etc.) vielmehr die Urinbefunde (Pyurie, Bakteriurie) zum Ausgangspunkte ihrer Untersuchungen gemacht $z u$ haben, ist zweifellos berechtigt. Denn gar häufig, wenn die Infection ohne besondere Symptome verläuft, ist eine strenge Localisirung und Rubricirung schwierig oder unmöglich. Man müsste denn in allen diesen Fällen zur Cystoskopie und zum Ureterenkatheterismus greifen, was mit der Belanglosigkeit vieler dieser Infectionen nicht im Einklang stehen würde. Wenn daher auch Rovsing den Begriff der ${ }_{n}$ Harninfection" (Guyon) nur als Rumpelkammerbezeichnung glossirt, so wird er 
vor der Hand aus Mangel an Besserem doch bestehen bleiben müssen. In allen solchen Fällen natürlich, in welchen deutliche Symptome auf eine bestimmte Erkrankung hinweisen, ist eine stricte Classification am Platze.

Das Capitel der Harninfectionen geht aus von der nunmehr sichergestellten Thatsache, dass der normale Harn des gesunden Individuum bakterienfrei ist (Guyon). Diese Erfahrung konnte letzthin noch Baisch durch das exacte Verfahren der sterilen Harnentnahme bei Laparotomien vermittels Blasenpunetion erhärten. Die früheren gegentheiligen Ansichten (Hofmeister u. a*) sind demnach als hinfällig zu bezeichnen und das Eindringen von Keimen in die Blase und die übrigen Harnwege - mitunter ganz bedeutungslos - als ein anormaler Zustand in den Bereich der Pathologie zu verweisen.

Die Erforschung der Harnkeime beschränkte sich bei der Mehrzahl der Autoren begreiflicherweise auf Urine bei offensichtlichen Harnerkrankungen. Nur wenige systematische Untersuchungsreihen liegen vor, von denen besonders die Befunde Albeck's für unsere Betrachtung interessant und wichtig sind.

Albeck konnte bei 150 unausgewählten Nichtschwangeren in 20 Fällen $=13$ pCt. pathogene Bakterien im Urin nachweisen, hierunter 14 mal das Bact. coli (also in 9 pCt. aller Fälle und in $70 \mathrm{pCt}$. der bakterienhaltigen Urine). Bei 392 Schwangeren fand er in 67 Fällen $=17$ pCt. Harnbakterien, hierunter 56 mal das Bact. coli (also in 14 pCt. aller Fälle und in 84 pCt. der bakterienhaltigen Urine): ferner bei 392 Gebärenden in 55 Fällen $=14 \mathrm{pCt}$, hierunter $43 \mathrm{mal}$ das Bact. coli (also in $11 \mathrm{pCt}$. aller Fälle und in 79 pCt. der keimhaltigen Urine).

Veranschaulichen wir uns diese Zahlen und die Werthe einiger anderer Autoren in einer Tabelle, so ergiebt sich folgendes Frequenzbild für das Bact. coli (siehe Tabelle V):

Aus dieser Tabelle geht zur Genüge die hohe Antheilnahme des Bact. coli an den Harninfectionen hervor. Die mehr oder weniger grossen Divergenzen in den Zahlenergebnissen der einzelnen Autoren hängen wohl z. Th. mit der verschieden häufigen Heranziehung des weiblichen Geschlechtes zusammen, welches aus den mannigfachsten Gründen mehr zur Coliinfection disponirt. Eine ausnehmend niedrige Zahl — dazu noch an Frauen gewonnen hat Baisch aufzuweisen. Er fand das Bact. coli nur in $25 \mathrm{pCt}$. 
Tabelle $\mathrm{V}$.

\begin{tabular}{|c|c|c|c|c|c|}
\hline Autor & $\begin{array}{l}\text { Gesammtzahl der } \\
\text { untersuchten Fälle }\end{array}$ & $\mid \begin{array}{c}\text { Bact.coli } \\
\text { pCt. }\end{array}$ & $\begin{array}{l}\text { Zabl der bakterien- } \\
\text { haltigen Urine }\end{array}$ & $\begin{array}{c}\text { Bact. coli } \\
\text { pCt. }\end{array}$ & Bemerkungen \\
\hline Albarran- & & & & & \\
\hline $\begin{array}{l}\text { Faltin } \\
\text { Foltin }\end{array}$ & - & - & $\begin{array}{l}\text { Du Harnurectionen } \\
86 \text { Cystitiden }\end{array}$ & $\begin{array}{l}47=94 \\
40=46\end{array}$ & 15 mat rem \\
\hline Brown & $\ldots$ & $\ldots$ & 100 acute 0 stitiden & $60=60$ & _- \\
\hline Suter & - & - & 200 Oystitiden & $83=41$ & - \\
\hline Melchior & - & - & 72 & $37=51$ & - \\
\hline her & - & - & ", & 70 & - \\
\hline Rostoski & - & - & & 80 & - \\
\hline Posner-Lewin & - & - & 22 & $11=50$ & 8 mal rein \\
\hline Baisch & - & - & $\begin{array}{c}40 \text { postoperative } \\
\text { Crstitiden }\end{array}$ & $10=25$ & $\begin{array}{c}\text { Misen- } \\
\text { infeetionen }\end{array}$ \\
\hline Albeck & 150 Nichtschwangere & $14=9$ & 20 Urininfectionen & $14=70$ & - \\
\hline$\%$ & 392 Schwangere & $56=14$ & 67 & $56=84$ & 55 mal rein \\
\hline " & 392 Gebärende & $43=11$ & 55 & $43=79$ & - \\
\hline$"$ & & - & $\begin{array}{l}92 \\
\text { (bei Gebärenden) }\end{array}$ & $79=86$ & $76 \mathrm{mal}$ rein \\
\hline
\end{tabular}

und immer in. Mischinfection ${ }^{1}$ ). Die postoperativen Cystitiden scheinen demnach eine Sonderstellung einzunehmen.

Wenn wir nunmehr unsere Resultate veröffentlichen, so sei vorausgeschickt, dass nur solche Fälle Berücksichtigung fanden, welche entweder durch die Menge der Leukocyten oder durch die Zahl der Bakterien zweifellose Zeichen einer Infection darboten. Einzelne Leakocyten im Urin sind bei Schwangeren und Wöchnerinnen häufig anzutreffen: desgleichen vereinzelte Bakterien, welche in der Regel aus der Urethra in die Blase durch den Katheter verschleppt sind. Gerade von letzterem Umstande konnten wir uns mehrmals überzeugen, indem nachgewiesene Urethralkeime (Bact. coli) aus dem Urin gezüchtet werden konnten, ohne dass Sediment- oder Trockenpräparate irgend welche abnormen Bestandtheile hatten erkennen lassen (ef. Fall 1--3 der casuist. Beiträge). Bei unseren gesammten Untersuchungen wurde darauf geachtet und fast ohne Ausnahme durchgeführt, dass der Urin sofort nach der Katheterentnahme zur Verwendung kam. Regelmässig wurde ein SedimentDeckglaspräparat und bei pathologischem oder zweifelhaftem Befunde ein gefärbtes Trockenpräparat angefertigt.

Unsere Untersuchungen führten zu folgenden Ergebnissen:

126 Schwangere (durchschnittlich in den 4 letzten Schwangerschaftsmonaten): 16 Harninfectionen $=12,5$ pCt.; darunter

1) In der Folgezeit änderte sich dann dieser Befund. 
2 mal Bact. coli $=1,5$ pCt. (also in 12 pCt. der bakterienhaltigen Urine). $16 \mathrm{mal}$ Pyurie $=12,5$ pCt.; $2 \mathrm{mal}$ Bact. coli (Reininfection) $=1,5$ pCt.; 14 mal andere Bakterien $=11$ pCt. (darunter 2 mal Gonokokken). 0 mal Bakteriarie $=0 \mathrm{pCt}$.

203 Wöchnerinnen: 59 Harninfectionen $=29$ pCt.; darunter 29 mal Bact. coli $=14$ pCt. (also in 50 pCt. der keimhaltigen Urine). $54 \mathrm{mal}$ Pyurie $=26,5 \mathrm{pCt} . ; 21 \mathrm{mal}$ Bact. coli (rein) $=10$ pCt.; 5 mal Bact. coli (Mischinfection) $=2,5$ pCt.; 28 mal andere Bakterien $=14$ pCt. (darunter 4 mal Gonokokken). 5 mal Bakteriurie $=2,5 \mathrm{pCt}$; 3 mal Bact. coli (rein) $=1,5$ pCt.; 2 mal andere Bakterien $=1 \mathrm{pCt}$.

41 Aborte: 7 Harninfectionen $=17$ pCt.; darunter 5 mal Bact. coli (also in $71 \mathrm{pCt}$. der keimhaltigen Urine). $6 \mathrm{mal}$ Pyurie $=14,5$ pCt.; 4 mal Bact. coli (rein) $=9,5$ pCt.; 2 mal andere Bakterien $=5$ pCt. 1 mal Bakteriurie $($ Coliinfection $)=2,5$ pCt.).

Aus unseren Befunden dürfen wir schliessen, dass Harninfectionen bei Wöchnerinnen viel häufiger als bei Schwangeren vorkommen (29 pCt. gegen 12,5 pCt.). Diese Frequenzsteigerung ist in erster Linie auf eine erhebliche Zunahme der Coliinfection im Wochenbett zurückzuführen. Während nämlich das Bact. coli bei Schwangeren nur 12 pCt. der Harninfectionen ausmachte, war es bei Wöchnerinnen in der Hälfte derselben anzutreffen. Leider fehlen in der uns vorliegenden Literatur ähnliche systematische Untersuchungen, welche zu Vergleichsstudien geeignet wären. Aus der Albeck'schen Arbeit lassen sich eben nur die bei Schwangeren erhobenen Befunde verwerthen, da wir bei Gebärenden (womit wohl Kreissende gemeint sind) keine besonderen Untersuchungen angestellt haben. Die Gesammtzahl der Harninfectionen bei Schwangeren giebt Albeck auf 17 pCt. an, eine Zahl, welche nur wenig von der unserigen abweicht $(12,5$ pCt.). Als sehr unterschiedlich erweist sich aber die beiderseits eruirte Betheiligung des Bact. coli (Albeck 14 pCt. bezw. 84 pCt., wir nur 1,5 pCt. bezw. 12 pCt.). Selbst wenn. wir noch 2 Urine hinzuaddiren - von denen der eine vorübergehend Colibakterien aufwies, bei einer am nächsten und einem späteren Tage angestellten Nachuntersuchung sie aber vermissen liess, von denen der andere, 
ante partum entnommen, ohne mikroskopisch festgestellte Veränderungen auf dem Nährboden Colikeime aufgehen liess, und welch beide Fälle im Wochenbett echte Coli-Harninfectionen darboten - , waren bei unserem Material Coli-Harninfectionen in der Schwangerschaft eine grosse Seltenheit. Hieraus wäre zu folgern, dass bei der relativen Häufigkeit von Sehwangerschaftspyelitiden die Blaseninfection bei Schwangeren leicht auf das Nierenbecken übergreift (cf. Abschnitt III) und hier die Vorbedingungen zur Ausbildung des Pyelitiszustandes antrifft. (Ureterencompression durch den schwangeren Uterus etc.). Thatsächlich hatte auch der eine von uns beobachtete Fall eine schwere Pyelitis im VIII. Schwangerschaftsmonat acquirirt; über das Schicksal der anderen Patientin (Colicystitis im III. Schwangerschaftsmonat) konnte nichts in Erfahrung gebracht werden.

Derartige vermuthungsweise gezogene Schlüsse bedürfen aber gesicherterer Grundlagen. Es bleibt weiteren Untersuchungen vorbehalten, diẹ Häufigkeit der Coliharninfection in der Schwangerschaft zu beurtheilen und zu entscheiden, ob die ron uns - im Gegensatz zu Albeck - gemachten seltenen Beobachtungen lediglich vom Zufall dictirt waren.

Mit unserer Behauptung, dass im Wochenbett eine erhebliche Zunahme der Harninfectionen eintritt, und dass diese Zunahme auf ein Plus an Coliinfectionen zurückzuführen ist, stehen wir auf festeren Füssen. Bei einer Gruppe von 80 Frauen, deren Urine in der Schwangerschaft und später im Wochenbett zur Untersuchung kamen, war der Urin $46 \mathrm{mal}$ bei beiden Untersuchungen normal. Bei 4 Frauen waren Reizungserscheinungen der Schwangerschaft im Wochenbett wieder verschwunden. Von den restirenden 30 Frauen erwarben 21 ihre Harninfection erst im Wochenbett. 12 mal wurde hierbei das Bact. coli angetroffen: $8 \mathrm{mal}$ in Reininfection.

Die gegenwärtigen Vorstellungen über den vermehrten Keimgehalt der weiblichen Urethra im Wochenbett, welchen wir hinsichtlich des Bact. coli noch erweitern konnten, legen es nahe, einen Theil unserer Harninfectionen dem Katheter zur Last zu legen. Leider gehen systematische Untersuchungen nicht ohne den zweischneidigen. Katheterismus ab, wenn man einwandsfreie Resultate erzielen will. So konnten wir denn auch bei einzelnen Fällen trotz aller aseptischer Cautelen die Harninfection dem Katheterismus auf dem Fusse folgen sehen. Ein deutliches Bild der Katheterinfection giebt eine Untersuchungsserie von 69 Frauen, welche am 4. und 
8. Wochenbetttage katheterisirt wurden mit dem Ergebnisse, dass $35 \mathrm{mal}$ an beiden Tagen normaler Urin angetroffen wurde, 4 mal die Infectionserscheinungen des 4. Tages am 8. Tage wieder zurückgegegangen waren, $20 \mathrm{mal}$ an beiden und $10 \mathrm{mal}$ nur am 8 . Tage inficirter Urin vorgefunden wurde. Der grösste Theil dieser letzten 10 Fälle muss jedenfalls mit dem Katheterismus in Zusammenhang gebracht werden. Von diesen 69 Frauen waren 22 auch noch ante partum katheterisirt worden. 9 derselben, welche hierbei ganz oder nahezu normalen Urin dạrboten, zeigten am 4. Wochenbetttage eine deutliche Harninfection. Auch ein Theil dieser Fälle gebört aller Wahrscheinlichkeit nach zur Kategorie der Katheterinfectionen. Ueber dieSchädigungen von Seiten des Katheters wird man, selbst wenn man häufig nur mit Möglichkeiten rechnet, nicht im Unklaren sein.

Wenn wir nunmehr nochmals auf die Schlusszahlen unserer Untersuchungen zurückkommen, so werden wir die für Wöchnerinnen gefundene Höhe der Harninfectionen (29 pCt.) für die nicht katheterisirten Fälle entschieden herabdrücken müssen, ohne deshalb aber unsere Folgerung einschränken zu brauchen, dass die. Harninfectionen im Wochenbett (gegenüber der Schwangerschaft) bedeutend häufiger sind. Die unsere besondere Aufmerksamkeit einnehmende Frequenzziffer der Coliinfectionen besteht aber wohl gleichfalls zu Recht, und wir constatiren daher, dass die Coliinfectionen die Hälfte aller Wochenbettharninfectionen ausmachen. Die übrigen Harnkeime waren in absteigender Reihenfolge: Staphylokokken, Bac. proteus, Streptokokken (meist in Mischinfection mit Staphylokokken) und schliesslich vereinzelt andere pathogene Bakterien. Erstgebärende waren etwas häufiger betroffen als Mehrgebärende. Merkwürdigerweise hatte der frisch gelassene Urin nicht nur in der überwiegendén Mehrzabl der Coliinfectionen, sondern auch bei den übrigen Wochenbettcystitiden (z. B. auch bei Bac. proteus) zumeist saure Reaction. Während aber der Coliurin dauernd sauer blieb, gingen die anderen Urine bald in Alkalescenz über. Diese Beobachtung bestätigt die kürzlich wieder betonte Annahme (Suter, Tanaka), dass eine alkalische Reaction kein Erforderniss für $\mathrm{Cy}$ stitis ist, sondern letztere auch mit saurem Urin entstehen und einhergehen kann, unabhängig davon, ob die Cystitiserreger den Harnstoff zersetzen oder nicht. Der "saure" Harn bei Coliurie ist schon immer als ein Characteristicum bezeichnet worden. Nur von einzelnen Autoren wurden Abweichungen von dieser Regel beschrieben. Baisch glaubt sie auf die Anwesenheit zahlreicher 
Leukocyten zurückführen zu können. Wir selbst hatten mehrmals Gelegenheit, neutrale oder alkalische Reaction des Coliurines zu beobachten, theils in vorbehandelten, theils in frischen Fällen. Irgendwelche Beziehungen der Reaction zur Eitermenge konnten wir jedoch nicht feststellen. Wir neigen eher zu folgender Annahme:

1. Die saure Reaction des Coliurines ist oft eine secundäre, hervorgerufen durch Lebensäusserungen des Bact. coli. Wit anderen Worten: Primär nichtsaurer (neutraler, alkalischer) Urin kann secundär durch das Bact. coli in sauren Harn umgewandelt werden. Beweis: Verimpfung von Urincolikeimen auf schwach alkalische Bouillon oder schwach alkalisirten sterilen Urin hat oft Sauerwerden dieser Substrate zur Folge.

2. In vielen Fällen ist die saure Reaction des Coliurines nativ (primär). Beweis: Die schwach alkalische Bouillon wird durch das Bact. coli (aus dem sauren Urin gezüchtet) in ihrer Reaction häufig nicht beeinflusst. Der betreffende Colistamm besitzt in solchem Falle also keine Säurerungsenergie und kann nicht die saure Reaction des Urins hervorgerufen haben.

3. War der Nierenurin neutral oder alkalisch, so kann sich der Blasenurin nach Obigem folgendermaassen verhalten:

a) der Blasenurin behält die neutrale oder alkalische Reaction bei, wenn nämlich das Bact. coli keine Säuerungsenergie besitzt. Solche Urine ändern auch nach längerem Stehen ihre Reaction nicht.

b) Der Blasenurin hat trotz Säuerungsvermögens des Bact. coli neutrale oder alkalische Reaction, wenn nämlich die Entleerung der Blase (Katheterismus) so schnell vor sich geht, dass dem Bact. coli wegen der Kürze der Zeit die Möglichkeit genommen ist, seine Säterungswirksamkeit zu əntfalten. Solche Urine werden nach einigem Stehen sauer.

c) Der Blasenurin wechselt seine Reaction und wird sauer, wenn das mit Säuerungsenergie ausgestattete Bact. coli Gelegenheit hat (seltenere Entleerung der Blase), in Wirksamkeit zu treten.

4. Alkalische Reaction des Coliurins ist immer auf native Alkalescenz und nie auf Alkalibildung durch das Bact. coli zurückzuführen.

Beweis: Impft man einen sauren sterilen. Urin mit solchen 
(aus alkalischem Coliurin gewonnenen) Colikeimen, so bleibt der Urin stets sauer.

Diese Ueberlegungen lassen es erklärlich erscheinen, warum man bei der Coliinfection fast immer sauren oder nur selten alkalisehen Urin antrifft. Wenn an sich schon der Urin physiologischer Weise sauer ist, so kann bei Alkalescenz desselben ( $\mathrm{z}$. B. nach reichlichem Genuss von Vegetabilien) die Reaction noch durch Kräfte des Bact. coli in die saure übergeführt werden. Aus Vorstehendem geht aber zugleich hervor, dass - bei der Variabilität des Bact. coli - von einer Gesetzmässigkeit der "sauren" Coliurinreaction nicht die Rede sein kann.

Der Geruch des Coliurins wird von den meisten Autoren als unangenehm oder übel, in einzelnen Fällen sogar als fäculent bezeichnet. Demgegenüber möchte nwir betonen, dass in den vielen Hunderten von Coliurinuntersuchungen wir fast stets faden Geruch, in einer kleinen Zahl üblen oder sogar fauligen, niemals aber fäculenten Geruch bemerkten.

Schlusssätze: Coliinfectionen sind in der Schwangerschaft viel seltener anzutreffen als im Wochenbett, wo sie die Hälfte aller Harninfectionen ausmachen. (Auch bei Aborten wird der Urin häufig mit Bact. coli inficirt.) Die Infection ist in einem Theile der Fälle dem Katheterismus zur Last zu legen.

Die Reaction des Coliurins ist meist sauer, der Geruch fade.

\section{Die Erkrankungsformen.}

\section{Specieller Theil.}

Unser gesammtes Material, welches über 100 Coliharninfectionen umfasst, zerfällt nach klinischen Gesichtspunkten geordnet in die 3 bekannten Hauptformen der Bakteriurie, Cystitis und Pyelitis. Wir werden darauf verzichten können, diese Krankheitsbilder, welche in vielen Publicationen der letzten Jahre eingehende Beschreibung gefunden haben, bis in ihre Einzelheiten zu verfolgen. Wie dic ganze Arbeit keinen Anspruch auf Vollständigkeit erheben will, wird auch in diesem Kapitel nur zu ganz besonderen Fragen Stellung genommen, welche in ihrer bisherigen Beantwortung entweder unsere Kritik herausforderten oder noch weiterer Mitarbeit zu bedürfen schienen. Anhangsweise werden dann noch einzelne uns charakteristisch erscheinende Beiträge verschiedenster Art angegliedert, welche mehr als theoretische Auseinandersetzungen unsere Behauptungen stützen und die Casuistik bereichern mögen. 
a) Die Bakteriurie.

Der von Roberts ${ }^{1}$ ) (1881) geschaffene Begriff der Bakteriurie wurde früher und wird auch heute noch als ein Krankheitsbild sui generis aufgefasst. Nach Krogius ${ }^{1}$ ) ist es charakterisirt einerseits durch das Vorhandensein zahlreicher Bakterien im frisch gelassenen Urin, andererseits durch das Fehlen ausgeprägter Symptome eines entzündlichen Processes in den Harnwegen (keine oder nur spärliche Leukocyten). Der Urin soll dabei stets sauer und gewöhnlich frei von Eiweiss sein und eine diffus getrübte, meist fade oder unangenehm riechende Flüssigkeit darstellen, deren Unklarheit sich beim Stehen nicht absetzt. Kornfeld hat versucht, das Krankheitsbild noch schärfer zu präcisiren, indem er die "reine genuine Form" " 0 den transitorischen Bakteriurien abgrenzte. Die reine genuine Affection ist nach seiner Ansicht ein specifisches Erzeugniss des Bact. coli, entsteht gewöhnlich durch directe Ueberwanderung des Keimes aus dem Darm, besitzt geringe T'endenz zum Ascendiren gegen die Nieren und ist niemals Vorstufe oder Endausgang einer Cystitis.

Bevor wir in eine Erörterung des Themas eintreten, scheint es uns zweckmässig, einige Beobachtungen, welche dem Befunde nach in die Kategorie der Bakterien fallen, vorauszuschicken.

\section{Coli-Bakteriurien.}

1. Frl. K. 23. 5. Neutral. Vereinzelte Erythrocyten und Leukocyten. Einige Epithelien. Massenhaft Colistäbchen.

2. Frl. St. 29. 5. Trüb, alkalisch. Reichljch Salze. Vereinzelte Leukocyten und Erythrocyten. Massenhaft Colistäbchen.

3. Frl. G. 7. 7. Sauer, trüb. Einzelne Leukocyten. Massenhaft Colistäbchen.

4. Frl. R. 16. 9. Sauer, trüb. Ganz vereinzelte Leukocyten. Gewimmel von Colistäbchen.

5. Fr. H. 11. 8. Sauer. Vereinzelte Leukocyten. Massenhafte Colistäbchen, z. Th. in Ketten. (Anschliessend heftige Colicystitis.)

6. Frl. G. 18. 8. Sauer. Ganz vereinzelte Leukocyten. Gewimmel von Colistäbchen. (Vorher Cystitisbefund.)

7. Frl. Bl. 22. 7. Sauer. Vereinzelte Leukocyten. Massenhaft Colistäbchen. (Vorher Cystitisbefund.)

8. Frl. P. 24. 8. Ganz vereinzelte Leukocyten. Massenhaft Colistäbchen. (Vorher und nachher Cystitisbefund.)

9. Fr. M. 26. 8. Sauer. Zahlreiche Colistäbchen; sonst o. B. (Vorher und nachher ausserdem zablreiche Leukocyten.)

10. Fr. R. 16. 7. Sauer. Zablreiche Colistäbchen; z. Th. in Ketten; sonst o. B. (Vorher geringe Menge Leukocyten.)

1) Cit. nach Kornfeld. 
11. Frl. W. 26. 6. Alkalisch. Sehr viele Colistäbchen, z. Th. in Ketten; sonst o. B.

12. Frl. St. 26. 6. Schwach sauer. Einzelne Colistäbchen; sonst o. B.

Nichtcoli-Bakteriurien.

1. Frl. L. 24. 7. Sauer. Vereinzelte Leukocyten. Massenbaft Stäbchen. (Proteus?)

2. Frl. Sch. 1.9. Neutral, opalescirend. Ganz vereinzelte Leukocyten. Gewimmel von grampositiven Stäbchen.

3. Fr. K. 1. 9. Schwach sauer, opalescirend. Vereinzelte Leukocyten. Sebr viele Epithelien. Viele Staphylokokken und grampositive Diplokokken. (Vorber ansserdem zahlreiche Leukocyten.)

4. Fr. U. 21. T. Sauer; fạulig. Wenige Leukocyten. Massenhaft Streptokokken.

5. Fr. U. 19. 8. Sauer; klar. Einzelne Leukocyten. Viele plumpe grampositive Stäbchen.

Derartige Befunde, welche allerdings $z$. Th. transitorischen Charakters sind, werfen ein eigenthümliches Licht auf das Wesen der Bakteriurie. Wir werden uns die Frage vorlegen müssen: Ist es statthaft, von einer "reinen genuinen" Form zu sprechen und werden die von Kornfeld an diese geknüpften Postulate in allen Punkten erfüllt? Was schon die Specifität der Coli-Ursächlichkeit anbelangt, muss man stutzig werden, wenn unter 17 Bakteriuriebefunden $5 \mathrm{mal}$ andere Bakterien angetroffen wurden. Wenn bisher nur vereinzelte Beobachtungen von Nichtcolibakterien gemacht wurden [Mellin ${ }^{1}$, Albeck], so mag dies in der geringen Anzahl systematischer Untersuchungen liegen. In Folge seiner Beharrlichkeit wird das Bact. coli zweifellos das grösste Contingent der chronischen Bakteriurien stellen. Es kam uns aber darauf an, zu zeigen, dass von einem coli-specifischen Symptomencomplex nicht die Rede sein kann. Die saure Reaction der Coliurien ist sicherlich die Regel, jedoch sind Ausnahmen nicht selten. Von unseren 12 Colibakteriurien zeigten 3 nichtsaure Reaction. Betreffs des Infectionsmodus neigt Kornfeld der Ansicht Wreden's und Reymond's za. Wenn er aber eine Beobachtung Melchior's (Frau, niemals katheterisirt, erkrankt plötzlich an Harnbeschwerden mit trübem Urin und den sonstigen für Bakteriurie charakteristischen Symptomen) als nungemein exact und zutreffend für den Befund einer echten, genuinen Bakteriurie" ansieht, so muss dem doch entgegengehalten werden, dass bei dem enormen Bakteriengehalt der weiblichen Urethra und der klinisch häufig genug erwiesenen

1) Cit. nach Stoeckel. 
Ascendenz der Keime die urethrale Infection niemals auszuschliessen, vielmehr die naheliegendste ist. Beim Manne mögen andere Verhältnisse mitspielen. Doch sind auch in der männlichen Urethra Colikeime (besonders nach überstandener Gonorrhoe) keine Seltenheit. Ferner ist nicht einzusehen, weshalb die Colikeime gerade bei der Bakteriurie vor den Ureteren Halt machen sollten, was doch ganz gegen ihre Gewohnheit wäre. Albeck hat denn auch (bei 7 Wöchnerinnen mit Bakteriurie) nachweisen können, dass je 3 mal beide bezw. 1 Harnleiter bakterienhaltig waren und nur 1 mal beide Ureteren sterilen Befund ergaben. Auch Mirabeau machte die Beobachtung, dass bei Bakteriurien "die Colibacillen schon in dem aus den Nieren abfliessenden Urin" vorhanden sind. Was schliesslich das hypothetische Unabhängigkeitsverhältniss der Bakteriurie zur Cystitis betrifft, so wird man sich, meine ich, wohl davor hüten müssen, aus einem zufällig aufgenommenen Befunde auch auf eine primäre Erkrankung zu schliessen. Bekanntlich geht die Colicystitis häufig ohne jegliche subjective Beschwerden einher, sodass die Betroffenen garnichts von ihrer Erkrankung zu wissen brauchen und daher unbewusst unrichtige anamnestische Angaben machen. Aus unseren Untersuchungen geht aber hervor, dass die Colibakteriurie sowohl ein Vorstadium wie auch ein Consecutivzustand der Colicystitis sein kann.

Indem wir uns somit in Gegensatz zu Kornfeld setzen, geben wir für die Colibakteriurie folgende Definition: Die Colibakteriurie stellt eine transitorische oder chronische Infection des Urins dar mit Keimen, welche keine pyogene Eigenschaften entfalten; sei es, dass sie an sich wenig virulent sind (primäre Bakteriurie), sei es, dass sie ihre Virulenz im Verlaufe der Infection eingebüsst haben (secundäre Bakteriurie als Folgezustand einer Cystitis), sei es schliesslich, dass die Vorbedingungen für eine Eiterbildung fehlen (normale, functionstüchtige Blase). In letzterem Falle tritt erst bei irgend welchen Störungen die Pyurie auf (secundäre Colicystitis). Die Colibakteriurie ist die häufigste Form aller Bakteriurien, giebt zumeist (nicht immer) saure Reaction und entsteht in der Regel durch Ascendenz der Keime aus der Urethra. Sie kann eine Vorstufe, ein Zwischenstadium und ein Endausgang einer pyogenen Coliinfection sein.

Die Diagnose der Colibakteriurie wird aus dem Urinbefund gestellt. Ihre Behandlung deckt sich im wesentlichen mit derjenigen der Colicystitis. 
b) Die Cystitis.

Eine strenge Sonderung zwischen Colibakteriurie und Colicystitis (Kornfeld) ist rom pathologischen Standpunkte zweifellos berechtigt, kliniseb jedoch nicht immer durchführbar und auch ohne Belang, da Symptome und Therapie keine principiellen Unterschiede aufweisen. Die Colicystitis geht immer mit Pyurie einher. Häufig finden sich Eiterkörperchen in grosser Menge im Urin, mitunter nur in geringerer Zahl, somit einen Uebergang zur Bakteriurie anbahnend. Das Verbältniss der Eitermenge zur Anzahl der Bakterien zeigt die gleiche Inconstanz. Einmal trifft man massenhaft Eiterzellen und nur wenig Bakterien, ein anderes Mal den umgekehrten Befund an. Das subjective Befinden ist meist nicht gestört; nur in einzelnen Fällen wurde über cystitische Beschwerden geklagt. Das Cystoskop, welches die schwereren Cystitiden ohne Weiteres erkennen lässt, versagt bei den milderen Formen. Gewöhnlich findet sich nur eine mässige Injection der Trigonumgefässe.

Die Diagnose ergiebt sich aus dem Urinbefund. Die grosse Mehrzahl der Colicystitiden gelangt wohl kaum zur Kenntniss des Arztes, wenn nicht gerade subjective Beschwerden oder sonstige Erscheinungen (trüber Urin) eine Urinuntersuchung veranlassen. Auffallend ist die starke Schmerzhaftigkeit des Katheterismus, welche selbst bei der Bakteriurie nur selten vermisst wurde und auf eine Mitbetheiligung der hinteren Urethra schliessen lässt.

Therapeutisch haben uns Collargolinstillationen derart gute Dienste erwiesen, dass wir uns früheren Empfehlungen anschliessen möchten. Das Collargol zeichnet sich vor dem Arg. nitr. und anderen' Silberpräparaten durch seine reizlosen Eigenschaften aus, weshalb die Patienten es meist anstandslos eine halbe bis ganze Stunde zurückbehalten können. Nachdem die Blase von Urinresten und sonstigen abnormen Bestandtheilen durch eine vorherige Borwasserspülung gereinigt ist, injiciren wir 100 g einer 1, proc. Lösung, zu Beginn der Erkrankung täglich, später seltener. Sind Mictionsbeschwerden vorhanden, fügt man der Collargollösung zweckmässig etwas Glycerin $(10 \mathrm{~g})$ hinzu, um die nach ea. einer halben Stunde angesetzte Entleerung der Blase zu erleichtern. Die Wirksamkeit der Collargolbehandlung konnten wir auch beim Dauerkatheterismus - dem Prüfstein der Therapie - erkennen, indem die sonst unvermeidliche Colinfection ausblieb. Man wird sofort nach Einführung des Danerkatheters mit der Behandlung beginnen müssen 
und erst vor der Entfernung des Katheters dieselbe beschliessen. Die letzte Collargolinjection (mit etwas Glycerin versetzt) soll also spontan ausgeschieden werden.

Ausser der Diät und der Verordnung grösserer Flüssigkeitsmengen erprobte sich uns bei der Collargolbehandlung vor allem das Salol in Gaben von 3 mal 1,0 täglich. In einzelnen Fällen sahen wir nach alleiniger Saloltherapie die Colicystitis schwinden, eine werthvolle Erfahrung, da wir ihr zufolge von dem Salol auch eine Einwirkung auf die in den Ureteren befindlichen Colikeime erwarten dürfen. Bei der häufigen Ascendenz der Colibakterien in die Ureteren ist aber gerade die Vernichtung dieser Keime wichtig.

Die Vereinigung der Collargol- und Saloltherapie erscheint uns daher als die aussichtsreichste Bekämpfung (und Verhinderung) der Coli-Harninfectionen. Durch sie wird die bis dahin schlechte Prognose in ein viel günstigeres Licht gestellt. In erster Linie wird man naturgemäss der Prophylaxe gerecht werden müssen. Nachdem wir in unseren systematischen Untersuchungen häufig genug die Schädigungen des Katheterismus (namentlich im Wochenbett) erkennen konnten, lassen wir jetzt, wenn alle anderen (bekannten) Maassregeln versagen, eine Wöchnerin lieber am ersten Tage post partum zum Zwecke des Urinirens aufsitzen oder gar aufstehen, bevor wir zum Katheter greifen.

\section{c) Die Pyelitis.}

Schwangerschaft und Wochenbett bilden eine Hauptdomäne der Colipyelitis. Man hat daran gedacht, die Pyelitis in diesen $\mathrm{Zu}$ ständen als ein besonderes, von den übrigen Pyelitiden abgegrenztes Krankheitsbild za betrachten. Das ist sicherlich nicht richtig; Schwangerschaft und Wochenbett bilden eben nur -- im wahren Sinne des Wortes - die Helfershelfer des Bact. coli, welches gewissermaassen immer auf der Lauer liegt, in pyogene Thätigkeit zu treten. Wo fände es aber hierzu bessere Gelegenheit, als in dem durch den Druck des schwangeren Uterus gestauten Ureterenurin, welcher Zustand (Ureterendilatation) in der ersten Leit des Wochenbettes fortbestehen kann oder durch Harnretention der puerperalen Blase wieder neu geschaffen wird! Erwiesen ist, dass gerade das Bact. coli die Ursache der meisten dieser Pyelitiden bildet - eine Thatsache, die mit dem schnellen Vordringen der Colikeime in die Ureteren in Zusammenhang zu bringen ist. In vereinzelten Fällen werden wohl auch Darmstörungen in der 
Gravidität und im Wochenbett zu beschuldigen sein (endogene Infection).

Wir sind in der Lage, über 28 Pyelitiserkrankungen berichten zu können, von denen 12 selbst beobachtet, 16 aus dem Journal gezogen sind. 24 dieser 28 Fälle waren reine Coliinfectionen; bei einem weiteren Falle wurde eine Spielart des Bact. coli (Milchzucker nicht vergährend) festgestellt (Prof. Dietrich). Einmal handelte es sich dann noch um eine Pyocyaneusinfection und zweimal ist das Bakterium nicht näher bestimmt worden. Von den 28 Pyelitiden entfielen 11 auf die Schwangersehaft (darunter die Pyocyaneuserkrankung) und 17 auf das Wochenbett (darunter die 2 NichtcoliPyelitiden). Sie betrafen

Erstgebärende: 20

in der Schwangerschaft . . . . 7

im Wochenbett . . . . . . 13

Mehrgebärende: 8

in der Schwangerschaft . . . . 4

im Wochenbett . . . . . . 4

Sie waren localisirt:

16 mal in der rechten Seite

5 mal in der linken Seite

7 mal in beiden Seiten.

Wenn doppelseitige Affection bestand, war gewöhnlich die rechte Seite zuerst befallen und der Process hier von grösserer Intensität.

Die Schwangerschaftspyelitiden setzten ein:

$\begin{array}{lllll}1 & \text { mal im } & 4 .-5 . & \text { Monat } \\ 1 & n & n & 7 . & \\ 1 & n & n & 8 . & \\ 3 & n & n & 8 .-9 . & n \\ 1 & n & n & 9 . & \\ 1 & n & n & 9 .-10 . & " \\ 3 & n & n & 10 . & \end{array}$

Spontane Frühgeburt erfolgte 5mal:

$1 \mathrm{mal}$ im 4.-5. Monat

$\begin{array}{lllll}1 & n & n & 6 . & \\ 1 & n & n & 7 .-8 . & \\ 1 & n & n & 8 .-9 . & \end{array}$

(Lues)

Die Beziehungen der Schwangerschaftspyelitis zum Partus waren folgende: 
2 mal Fieberabfall ohne Geburtseintritt,

1 "Fieberabfall $1 \mathrm{Tag}$ vor Geburtseintritt,

1 "Blasensprung mit Fieberabfall, dann Stillstand der Geburt,

2 , Fieberabfall sofort nach Entbindung;

4. "Fortbestand der Erkrankung nach Entbindung und zwar 1 mal 2 Tage

$$
\begin{array}{llll}
1 & n & 4 & n \\
1 & n & 6 & n \\
1 & n & 7 & n
\end{array}
$$

Die Wochenbettpyelitiden setzten ein:

$1 \mathrm{mal}$ am 1. Tage

$$
\begin{aligned}
& 2 " n 2 . " n \\
& 3 n \text { " } 3 . \\
& 1 n n 4 \text {. } n \\
& 1 n \pi 6 . n \\
& 2 n " 7 ., n \\
& \text { (1" } " \text { 8. } " \text { im Anschluss an Ureterenkatheterismus) } \\
& 1 n, 12 . \\
& 1 n, 16 ., \\
& \text { (1 " "36. " im Anschluss an Entfernung des Dauer- }
\end{aligned}
$$

Aus diesen Einzelbetrachtungen dürfen wir wohl folgende Schlüsse ziehen:

1. Die Erkrankung trifft in der grössten Mehrzahl der Fälle Erstgebärende: eine Thatsache, die ungezwungen mit den vermehrten Jarnbeschwerden und -behinderungen in Zusammenhang zu bringen ist.

2. Das rechte Nierenbecken wird ungleich häufiger befallen Is das linke.

3. Die Schwangerschaftspyelitis ist in der Regel eine Errankung der letzten Monate; die Wochenbettpyelitis gewöhnlich ine Complication der ersten Woche.

4. Spontane Frühgeburt tritt in einem grossen Theile der chwangerschaltspyelitiden ein.

5. Der Eintritt der Wehen und des Partus hat in einem heile der Fälle Fieberabfall zur Folge, bei Fortbestehen der Errankung erstreckt sich das Fieber meist nur auf die ersten Wochensttage.

Unsere Beobachtungen stimmen im Allgemeinen mit denen ıderer Autoren überein. Nor hinsichtlich des häufigeren Betroffen- 
seins Erstgebärender werden auch gegentheilige Angaben gemacht [Ruppaner, Cathala 1 )]. Die auffallende Bevorzugung der rechten Scite knüpft eng an die Frage der Harnleitercompression (durch den schwangeren, rechtsgedrehten Uterus) an, für welche namentlich Sippel und Opitz eingetreten sind. Andere wiederum (Mirabeau, Orlowski) lehnen diese Erklärung ab und wollen mehr die Schwangerschaftsveränderungen der Blasenschleimhaut mit ihren wulstigen Vorsprüngen am Ureterostium beschuldigen. Die häufige rechtsseitige Affection führt Mïrabeau, ein Anhänger der endogenen Infectionstheorie, auf die innigen Beziehungen zwischen rechter Nicre und Darm zurück. Die wenigen Erfahrungen, die wir sammeln konnten, sprechen entschieden gegen die Deductionen Mirabeau's (abgesehen von unserer abweichenden Meinung über den Tnfectionsweg). Wohl war nach dem Eindringen des Katheters in das Ureterostium ein gewisser Widerstand zu überwinden, was wir manchmal auf eine straffere Spannung des leistenartig vorspringenden Lig. interuretericum beziehen durften; jedoch erfolgte der (continuirliche) Abfluss des gestauten Urins immer erst nach weiterem Vorschieben des Katheters. So stiess bei einer Schwangeren mit rechtsseitiger Pyelitis der Katheter in einer Höhe von $16 \mathrm{~cm}$ an und konnte erst nach Lagerung der Pat. auf die linke Seite (spielend) weitergeschoben werden. Hierauf Entleerung von ea. $50 \mathrm{ccm}$ eines trüben, eiter- und colihaltigen Urins. Da die Ureterendilatation nicht an einem Tage (Partus), vielmehr ganz allmählich sich zurückbilden kann, somit also eine gewisse Harnstauung in den Ureteren sich in die ersten Tage des Wochenbettes fortsetzt, findet auch die häufige rechtsseitige Wochenbettpyelitis hierin eine ungezwungene Erklärung.

Symptome und Diagnose: Die Symptomatologie der Pyelitis ist heut zu Tage hinlänglich bekannt. Wir haben den acuten foudroyanten, mit Schüttelfrösten eingeleiteten Beginn und die mehr einsehleichende chronische Form, die Neigung zu Recidiven und andere Eigenthümlichkeiten kennen gelernt. Aber trotzdem mag auch heute noch in einzelnen Fällen. den erfahrenen Kliniker eine gewisse Unsicherheit überfallen, wenn er zwischen Pyelitis und Appendicitis, Cholecystitis oder Sepsis zu entscheiden hat. Das chamäleonartige, überaus weehselvolle Verhalten des Bact. coli spiegelt sich auch in den durch dasselbe verursachten Erkxan-

1) Cit. nach Ruppaner. 
kungen wieder. Die Diagnose der Pyelitis ist darum häufig so schwierig, weil gerade dio charakteristischen Symptome fehlen können.

Folgende vier Kriterien, die wir als die Cardinalsymptome der Colipyelitis bezeichnen möchten, scheinen uns für die Diagnose entscheidend zu sein:

1. Der Kopfschmerz. Er fehlt fast nie und ist mitunter so heftig, dass er das ganze Krankheitsbild beherrscht. Gern treten die Cephalalgien schon einige Tage vor Beginn der Erkrankung auf, die Pyelitis somit gewissermaassen ankündigend.

2. Die Puls-Fiebercurve. Schon Opitz und in noch überzeugenderer Weise Lenhartz haben auf eine merkwürdig geringe Alteration des Pulses, auf einen Contrast $z$ wischen Puls- und Fiebercurve die Aufmerksamkeit gelenkt. Dieses Symptom, sowie das erstere, bezeugen, dass das Pyelitisfieber in der ersten Linie ein Resorptionsfieber (Colitoxine) ist. Nicht immer hält sich der Puls auf niedriger Höbe. Namentlich im Wochenbette, welches leicht zu Complicationen von Seiten des Genitaltractus neigt (z. B. Lochienstaung in Folge Blasenüberfüllung etc.), kann es zu einer Steigerung der Pulszahl und damit zu einer Verschleierung der typischen Curve kommen. Andere intercurrirende Krankheiten, starke Idiosynkrasie gegen das Coligift, nervöse Tachykardie, leichte Erregbarkeit etc. haben den gleichen Effect. In der Hälfte unserer Fälle hatten wir jedenfalls eine ganz charakteristische Curve. Einige Beispiele, zum Theil eine Ergänzung der Lenhartz'schen Mittheilung, mögen das typische und atypische Verhalten des Pulses beleuchten. (Tafel IX.)

3. Der Nierenschmerz. Meist klagen die Frauen schon zu Beginn der Erkrankung über dumpfe oder mehr stechende Schmerzen in der betroffenen Bauchseite oder Lendengegend. Ist der Spontan- oder Druckschmerz sehr heftig, so wird eine genaue Abtastung nicht immer möglich sein, zumal bei vorgeschrittener Schwangerschaft, welche an sich eine exacte Untersuchung erschwert In solchen Fällen ist vor Allem auf die Druckempfindlichkeit der betreffenden Lenden- (Nieren-) gegend (handbreit neben der Wirbelsäule im Bereiche der 12. Rippe) Gewicht zu legen, welche direct auf eine Nierenaffection hinleitet. In den meisten Fällen konnte bei combinirter Untersuchung der schmerzhafte untere Nierenpol palpirt oder die Druckempfindlichkeit ziemlich genau auf die Hilusgegend und den oberen Abschnitt des Ureters localisirt werden. 
Dass der Mc. Burney'sche Punkt häufig mitbetroffen ist, erklärt sich aus dem Verlaufe des Ureters. Die differentialdiagnostische Beurtheilung gegenüber der Appendicitis liegt hier in der Steigerung der Schmerzhaftigkeit nach der Nierengegend $z u$, in dem Lendenschmerz und ferner auch in dem Fehlen der Muskelspannung (défense musculaire). Schwieriger kann die Abgrenzung gegen Cholecystitis (Cholelithiasis) werden, besonders wenn sonstige dieser Erkrankung eigene Symptome (Icterus etc.) nicht hervortreten. Man wird berücksichtigen müssen, dass Gallensteine mehr anfallsweise auftretende Koliken verursachen, während die Pyelitis einen continuirlichen dumpfen Schmerz zu bereiten pflegt. Dabei localisirt sich die grösste Schmerz- und Druckempfindlichkeit bei ersterer Affection mehr an der vorderen Bauchseite unter dem Rippenbogen, von hier in die Tiefe nach dem Rücken ausstrahlend; bei der Pyelitis dagegen intensiver in der Rückengegend. Es wäre aber verfehlt, aus dem subjectiven Befunde der Schmerzen allein - in zweifelhaften Fällen -- die Diagnose aufbauen zu wollen. Hier sind naturgemäss auch (gleichwie bei Appendicitisverdacht) die anderen Kriterien zu Rathe zu ziehen, namentlich der Harnbefund (Pyurie auf der einen Seite - normaler Urin auf der anderen Seite; Gallenfarbstoffprobe etc.). Es bedarf noch der Erwähnung, dass die Nierenschmerzen und Druckempfindlichkeit zuweilen gänzlich vermisst werden. Das sind aber - bach unserer Erfahrung leichte Erkrankungen, welche gewöhnlich bald wieder zur Norm übergehen.

4. Der Harnbefund. Der Katheterismus, ein unbedingtes Erforderniss zur Sicherung der Diagnose, liefert einen trüben, meist sauren Urin mit zahlreichen Eiterzellen und Colistäbchen. Ausser Epithelien, vereinzelten Erythrocyten trifft man in einzelnen Fällen auch Fibringerinnsel, welche ganz mit Bakterien durchsetzt sind, an (Pyelitis membranacea). Bei schwereren Affectionen kommen mitunter auch Bakteriencylinder zur Beobachtung. Eine Trennung zwischen Cystitis- und Pyelitisharn konnten wir nicht durchführen, die von einzelnen Autoren angegebenen Kriterien (Rosenfeld) liessen zu häufig im Stiche. Ner Harnbefund ist daher nie allein, sondern nur im Verein mit den anderen Symptomen für die Diagnose verwerthbar. Die Entscheidung, ob die Niere in Mitleidenschaft gezogen (Pyelonephritis), ist meist möglich, mitunter aber erschwert, da einerseits sich granulirte Nierencylinder unter der Unmenge von Leukocyten verstecken können, andererseits der an sich schon opa- 
lescirende Coliurin bei der Eiweissprobe eine lejehte Trübung leicht übersehen lässt. Des Oefteren konnten wir in Arbeiten, die sich mit der Pyelitis beschäftigen, den Hinweis finden, dass, bei vollkommener Occlusion des betroffenen Ureters der Urin ganz normalen Befund zeigen könne. Diese Beobachtung machten wir nie. Bei der ascendirenden Form kommt ein derartiger negativer Befund wohl kaum in Betracht, und bei der seltenen endogenen Infection sollte man annehmen, dass vor der totalen Verschliessung des Ureters genügend Bakterien in die Blase gelangt sind, um den darin befindlichen Urin $z u$ inficiren. Eiterkörperchen wird man allerdings bei solchen (Ausnahme-) Füllen event. vermissen können.

Darf man also jedem einzelnen der vier Symptome eine $a b$ solute Zuverlässigkeit nicht zusprechen, so wird doch durch das Zusammentreten derselben die Sicherheit der Diagnosenstellung nicht lejden. Wir selbst beobachteten ungefäbr in der Hälfte unserer Fälle schon zu Beginn der Erkrankung den ganzen vierfachen Symptomencomplex, während er sich bei den übrigen meist im Verlaufe der Krankheit einstellte. Aus der Unsicherheit der einzelnen Symptome auf die Möglichkeit des Fehlens aller Erscheinungen zu schliessen, wäre nicht angängig. Ist z. B. in Folge Ureterenverschlusses der Urinbefund vollständig normal — wenn wir einen derartig extremen Fall annehmen wollen -, so muss ein Urinstauung im Nierenbecken vor sich gehen, d. h. wir werden neben einem Palpationsbefunde Schmerz- und Druckempfindlichkeit ete. vorfinden. Wenn man sich daran gewöhnt hat, bei unklaren fieberhaften Zuständen in der Schwangerschaft und im Wochenbette immer an die Möglichkeit einer Pyelitis zu denken (Olshausen), so wird man diese Erkrankung nicht leicht übersehen und über die Häufigkeit derselben erstaunt sein.

Es ist noch nachzutragen, dass zu Beginn der Erkrankung meist eine Verminderung der Harnmenge beobachtet wird. Desgleichen kann der Nachweis von Bact. coli im Blute - ein allerdings seltener Befund - werthvoll für Diagnose und Beurtheilung des Falles sein. Leukocytenzählung führt im Allgemeinen zu keinem Ergebnisse, kann jedoeh gegebenen Falls differentialdiagnostisch in Betracht kommen. Zum Beispiel würde eine geringe Zahl von Leukocyten, wenn Appendicitis in Frage steht, mehr für Pyelitis sprechen.

Gelegentlich mag es vorkommen, dass trotz aller diagnostischer Kunst eine befriedigende Klarbeit über das Wesen der Krankheit 
nicht erzielt werden kann. In solchen - wohl seltenen - Fällen tritt die Cystoskopie in ihr Recht. Sie demonstrirt uns ad oculos etwaige Veränderungen der Blasenschleimhant und belehrt uns zugleich über die Beschaffenheit des aus den Ureteren spritzenden Urins. Trüber eitriger Harn zeugt von einer Erkrankung in den oberen Harnwegen, lässt aber zugleich den Schluss zu, dass der Harnleiter durchgängig ist. Gänzlicher Mangel von Urinsecretion spricht für Verschluss des betreffenden Ureters. Für solche Fälle kommt der Ureterenkatheterismus in Frage -, wohl mehr aus therapeutischen als diagnostischen Gründen. Dieser Eingriff verlangt entschieden eine stricte Indication, wie eine jede Operation und sollte nicht einer "überflüssigen Sicherheit halber"s (Rovsing) angewandt werden. Wie leicht aus einer uncomplicirten Nierenbeckeninfection das schwere Bild der acuten Pyelitis heraufbeschworen werden kann, zeigte uns eine lediglich zur Completirung einer systematischen Untersuchung ausgeführte Ureterenkatheterisation, welcher die mit Schüttelfrost eingeleitete Attaque innerhalb weniger Stunden folgte (Temperaturabfall nach einem Tage). Immerhin ist die Cystoskopie und eventuell der Ureterenkatheterismus das souveräne Mittel, welches -- wenn die übrigen Kriterien nicht genügenden Aufschluss geben -- und der Verdacht auf andere schwere Erkrankungen (Appendicitis, Cholelithiasis etc.) die Sicherstellung der Diagnose verlangt, -- unter allen Umständen über die Nierenbecken Klarheit verschafft.

Therapie und Prognose: Da die Pyelitis gravidarum et puerperarum in der Regel in der aeuten Form auftritt, wird man zumeist mit conservativen Maassnahmen auskommen können. Lagerung auf die gesunde Seite (Seitenbauchlagerung), grosse Flüssigkeitsgaben (Milch, Thee) und Salol ${ }^{1}$ ) (3 mal 1,0 tgl.), Hochstellung des Kopfèndes des Bettes (zur Erleichterung des Urinabflusses), Regelung der Darmthätigkeit führten in unseren Fällen fast immer zum Ziele ${ }^{2}$ ). Das Fieber lief dann gewöhnlich in

1) Mit dem in erster Zeit verwandten Methylenblau (3 mal 0,5) hatten wir zweifellos gute Erfolge, wenn sie auch nicht der Salolwirkung gleichkamen. Abgesehen von der bei Urinuntersuchungen lästigen Blaufärbung des Harnes bekamen die Patienten nach einigem Gebrauche leicht Abscheu and Brechreiz. Hingegen wurde das Salol lange Zeit hintereinander gut vertragen.

2) Collargolblasenspülungen warden nuz bei Blasenbesehwerden (Dysurje etc.) gemacht, um Störungen der Function (Urinretention) zu verhindern. Im Allgemeinen ist die Collargoltherapie erst nach Ablauf der Pyelitis angezeigt. 
wenigen Tagen ab. Recidive waren mit dieser Therapie nicht immer $z u$ vermeiden, kamen jedoch nur in einem kleinen Theile der Erkrankten zur Beobachtung. In recidivirenden und sich hinschleppenden Fällen ist der Ureterenkatheterismus mit nachfolgender Nierenbeckenwaschung - wir verwenden wieder 1 proc. Collargol am Platze, wabei man aber mitunter nur Augenblickserfolge zu verzeichnen hat. Der Ureterenkatheterismus erscheint uns ferner immer angezeigt, wenn vorzeitige Wehen einsetzen, um damit wenigstens den Versuch zu machen, die drohende Frühgeburt aufzuhalten. Die künstliche Unterbrechung ist wohl meistentheils zu umgehen. Eine principielle Verwerfung derselben (Stoeckel) scheint uns aber nicht immer gerechtfertigt. Wir selbst waren in einem Falle vor die Frage der artificiellen Frühgeburt gestellt, als bei einer schweren Pyelitis dextra die Entzündung auch auf das linke Nierenbecken übersprang. Versagt in einem derartigen Falle, in welchem ein wesentlicher Theil der Therapie, die Seitenlagerung, fortfällt, der Ureterenkatheterismus, sind die Schmerzen heftig, das Fieber hoch und hilft sich die Natur nicht selbst durch Eintretenlassen von Wehen, so ist nach unserer Meinung doch ernstlich an die künstliche Unterbrechung zu denken. Wir geben zu, dass solche Fälle Ausnahmen sind und mochten betonen, dass wir bisher niemals den Eingriff auszuführen brauchten, die Möglichkeit einer directen Indication kann aber wohl nicht kurzweg abgelehnt werden.

Die chronischen Pyelitiden, primär oder im Anschluss an die acute Form entstanden, erheischen, falls die länger fortgesetzten conservativen Methoden ohne Erfolg bleiben and der Allgemeinzustand beängstigend wird, eine chirurgische Intervention (Nephrotomie etc.). Im Allgemeinen wird man aber in der Schwangerschaft und im Wochenbett ohne ehirurgischen Eingriff auskommen: Die Prognose der Schwangerschafts- und Wochenbettspyelitis ist bei richtiger Behandlung quoad vitam et restitutionem günstig. Es ist schon oben darauf hingewiesen worden, dass die Colibakterien das Gewebe selbst wenig alteriren oder garnicht schädigen. Daher läuft die Erkrankung meist in kurzer Zeit ab. Man wird zwar nicht an den 3 oder 4 bekannt gewordenen Todesfällen vorübergehen dürfen, man wird immer mit der Möglichkeit eines Relapses rechnen müssen: die Mehrheit der Colipyelitiden gibt aber sicherlich eine günstige Prognose. 


\section{Casuistische Beiträge.}

a) Einfacher Import von Bact. coli in die Blase ohne nachfolgende Blaseninfection.

1. Hausschwangere E. Bü. (Tab. 4: Hausschwangere No. 2). Ürin (19. 7.): hell, klar, sauer. Viele Epithelien, sonst o. B. Culturell: negativ.

2. Hausschwangere C. Bie. (Tab. 4: Hausschwangere No. 4). Urin $(27,5$.$) : hell, klar, sauer, o. B. Culturell: negativ.$

3. Hausschwangere E. KI. (Tab. 4: Hausschwangere No. 5). Urin (22. 7.) hell, klar, saver. O. B. Culturell negativ.

Urine am 16. 8. (8. Untersuchungstag der Tab. 4):

1. hell, klar, sauer. Einzelne Leukocyten, sonst o. B.

2. hell, klar, sauer, o. B.

3. hell, klar, sauer, o. B.

In allen 3 Fällen waren (vor der Urinentnahme) im Vestibularund Urethralsecrete Colibakterien nachzuweisen (s. Tabelle).

Bei allen 3 Urinen gingen auf dem Drigalski-Nährboden einzelne Coli-Colonien auf.

Nachuntersuchung am 21. 8.:

Urii 1: hell, klar, sauer, o. B.

Urin 2: hell, klar, sauer, einzelne Epithelien und Leukocyten.

Urin 3: hell, klar, sauer, einzelne Epithelien.

Drigalski: In allen 3 Fällen steril.

b) Blaseninfection nach aseptischem Katheterismus im Wochenbett.

4. Fr. A. A. 39 Jahre. IV p. Katheterismus am 4. Wochenbetttage (systematische Untersuchung).

24. 8. Urin: hell, klar, sauer, o. B. Cultnrell: negativ. Im Vestibular- und Urethralsecret zahlreiche Colibakterien nachweisbar.

28. 8. Urin: sauer; opalescirende Trübung; fader Geruch. Viele Leukocyten. Gewimmel von Colistäbchen.

30. 8. Urin: sauer, diffuse Trübung. Viele Leukocyten und Epithelien, Gewimmel von Colistäbchen.

c) Primäre Colicystitis (cf. Fälle 4, 13).

5. Fr. B. M. 28 Jahre. IVp. Eklampsie. Vaginaler Kaiserschnitt. 31. 8. Dauerkatheter. $100 \mathrm{~g}$ Collargol (1 pCt.)

1. 9. Urin: hell, klar, sauer. Cylinder. Albumen. Ganz vereinzelte Lenkocyten und Stäbchen, Drigalski: Bact. coli (1 Colonie). $100 \mathrm{~g}$ Collargol ( $1 \mathrm{pCt}$.).

2. 9. Urin: hell, klar, sauer. Vereinzelte Leukocyten und Stäbchen. Drigalski: Einzelne Coli-Colonieen gewachsen. $100 \mathrm{~g}$ Collargol (1 pCt.). Dauerkatheter entfernt.

3. 9. Urin: hell, klar, sauer. Viele Leukocyten. Keine Bakterien.. Drigalski: steril. $100 \mathrm{~g}$ Collargol.

4. 9. $100 \mathrm{~g}$ Collargol.

5. 9. Urin: bell, klar, sauer. Einzelne Leukocyten, sonst 0. B. Keine Bakterien. $100 \mathrm{~g}$ Collargol.

7. 9. Urin: Albumen in Spuren. Sonst nicht untersucht. Kein Collargol mehr. Starker, übelriechender Ausfluss. 
9. 9. Urin: hell, klar, sauer. Ein granulirter Cylinder. Einzelne Leukocyten, sonst 0 . B.

11. 9. Urin: hell, klar, sauer, o. B. Albumen in Spuren.

13. 9. Urin: hell, klar, sauer. Einzelne byaline Cylinder. Einzelne Epithelien und Erythrocyten. Zablreiclie Leukocyten und viele gramnegative Stäbchen. Culturell: Bact. coli (Reininfection).

d) Primäre Colicystitis (nach Dauerkatheterismus). (cf. Fälle im Text und Fälle 11, 14).

6. Frl. M. Ki. 24 Jahre. Ip.

16. 4. Ischuria paradoxa p. part. Dauerkatheter.

19. 4. Reichlich Eiterzellen, massenhaft Colistäbchen. katheter.

7. Fr. M. Tsch. 20 Jahre. Ip. Eklampsie. Kaiserschnitt. Dauer-

28. 5. Urin: Massenhaft Cylinder. Albumen $+(15 \mathrm{pCt}$ ), sonst o. B. Culturell: negativ.

29. 5. Urin: Gleicher Befund. Weniger viel Albumen.

1. 6. Dauerkatheter ausgestossen.

2. 6. Urin: trübe. Massenhaft Leukocyten und Colistäbchen.

e) Secundäre Coljeystitis (durch Dauerkatheterismus).

8. Frl. H. B. 21 Jahre. Ip.

9. 9. Klagt seit Partus (7. 9.) über erschwertes Uriniren. Hat seit gestern Abend noch nicht urinirt. Dauerkatheter. Urin: trüb, sauer. Massenhaft Eiterzellen und Staphylo- und Streptokokken.

11. 9. Urin: trüb, sauer. Massenhaft Eiterzellen und Colistäbchen. Ganz vereinzelte Staphylo- und Streptokokken.

14. 9. Dauerkatheter entfernt. Uriniren nachher ohne Beschwerden.

20. 9. Urin: trüb, saner. Zablreiche Eiterzellen und massenhaft Colistäbchen. (Reininfektion.)

$$
\text { f) Colibakteriurie (primär?). }
$$

9. Fr. B. W. 19 Jahre. Igrav. Abort.

26. 6. Urin: alkalisch. Sehr viele gramnegative Stäbchen, z. Th. in Kettes. Sonst $n$. B. Culturel]: Bact. coli. (Einzige Lntersuchung.) 10. Fr. M. R. 38 Jahre. XlV grav. Abort.

23. 6. Urin: sauer. Geringe Menge von Leukocyten. Viele Fpithelien. Selur viele Colistäbchen.

6. 7. Urin: sauer, hell. Zahlreiche Stäbchen, z. Th. in Ketten. Sonst 0. B. Culturell: Bact. coli.

g) Secundäre Colibakteriurie (nach Colicystitis).

11. Frl. H. Bl. 22 Jahre. Ip. Eklampsie. Dauerkatheter am 8. 7.

13. 7. Zahlreiche Leukocyten. Gewimmel von Colistäbchen.

19. 7. Dauerkatheter entfernt.

20. 7. Urin: saner; leicht trüb. Ganz vereinzelte Leukocyten und Epithelien. Massenhaft Colistäbchen. (Reininfektion.) 20. 7. Ganz vereinzelte Leukocyten. Massenhaft Colistäbchen.

12. Frl. M. G. 20 Jahre. Ip. Partus 6. 8.

9. 8. Urin: sauer, trüb. Massenhaft Leukocyten und Colístäbchen.

16. 8. Scbwach sauer. Geringe diffuse Trübung. Ganz vereinzelte Leukocyten. Gewimmel von Bact. coli. 


\section{h) Colicystitis (nach Colibakteriurie).}

13. Fr. E. H. 31 Jahre. Ip. Partus: 6. 8. Urin (i. partu): hell, klar, saner, o. B. Drigalski: steril.

9. 8. Urin: sauer, trüb. Einzelne Erythrocyten und Leukocyten. Sonst o. B. Drigalski: steril.

11. 8. Sauer. Vereinzelte Leukocyten. Massenhaft Stäbchen, z. Th. in Ketten. Culturell: Bact. coli. (Reininfection.)

16. 8. Schwach sauer, trüb. Massenhaft Eiterzellen und Colistabchen. (Reininfection.)

\section{j) Ascendirende Colipyelitis.}

14. Frl. M. H. 21 Jahre. Ip. Pubiotomie am 3. 4. Dauerkatheter.

12. 4. Massenhaft Eiterzellen und Colistäbchen.

21. 4. Reichlich Eiterzellen und Stäbchen, z. Th. in Haufen. Cnlturell: Bact. coli.

8. 5. Entfernung des Dauerkatheters (Vormittags). Bis zum Abend hat Patientin nicht urinirt. Blase stark gefüllt. Abendtemperatur $38,5^{\circ}$. Schmerzhaftigkeit und starke Druckempfindlichkeit der rechten Nierengegend (Fiebercurve No. 5).

15. Fr. M. R. 22 Jahre. II p. Partus 12. 5.

21. 5. Patientin leidet seit Partus an erschwertem Uriniren (nur bei starkem Pressen). Heute starke Kopfschmerzen und Fieber (Fiebercurve No. 11). Urin: Massenhaft Eiterzellen und Colistäbchen.

22. 5. Heftige Schmerzen in der rechten Nierengegend; daselbst starke Druckempfindlichkeit. Sehr heftige Kopfschmerzen.

16. Frl. Fr. Scb. 21 Jahre. Ip. Partus 25. 9.

26. 9. Blase bis Nabelhöhe gefüllt.

27. 9. Fieber (Fiebercurve No. 6). Starker Druckschmerz der rechten Niere. Urin: trüb, dunkelgelb, neutral. Massenhaft Leukocyten und Colistäbchen. (Reincultur.)

\section{k) Descendirende Colipyelitis (?).}

17. Frl. A. M. 24 Jahre. IVp.

6. 4. Partus ausserhalb, stehend in der Küche. Seit 8 Tagen keine Stuhlentleerung. Temperatur: $40,3^{\circ}$. Schüttelfrost. Sepsisähnlicher Zustand,

7. 4. Auf Einlauf und Ricinusgaben erfolgte 7 malige reichliche übelriechende Entleerung.

8. 4. Druckempfindlichkeit der rechten Niere, Urin: Albumen + $(1 / 4 \mathrm{pCt}$.). Vereinzelte Erythrocyten und Epithelien. Zahlreiche Leukocyten und massenbaft Colistäbchen.

Anmerkung bei der Correctur: Die Verhandlungen der deutschen Gesellschaft für Urologie (II. Congress, Berlin 19. bis 22. April 1909) erschienen erst nach Abschluss dieser Arbeit im Druck. Es konnte daher zu den interessanten Referaten von v. Frisch u. Barth über die eitrigen, nicht tuberculösen Affectionen des Nierenbeckens und der Niere nicht Stellung genommen werden. Zum grossen Theile decken sich unsere Ansichten mit den dort vertretenen. Namentlich in der Forderung einer stricten 
Indication für die Ureterensondirung bei Pyelitis befinden wir uns in Uebereinstimmung mit v. Frisch. Bezüglich des Infectionsmodus schreibt v. Frisch dem endogenen Weg grössere Bedeutung zu als wir ihm zuerkennen möchten. Was speciell die Coliinfectionen anbelangt, meint $v$. Frisch in seinen Schlussfolgerungen: Ueber die Ursachen der grossen Häufigkeit des Bact. coli als Infectionserregers, sowie über die Wege, auf welchen dasselbe in das Nierenbecken gelangt, erscheinen weitere experimentelle Arbeiten unter steter Berücksichtigung der klinischen Erfahrungen erwünscht.

\section{Literatur.}

(Nur die der Arbeit vorgelegenen Originalien und Referate finden Erwähnung; die übrige gesammte Literatur ist daselbst einzusehen.)

1. Albeck, Balkteriurie und Pyurie bei Schwangeren und Gebärenden. Zeitschrift f. Gyn. Bd. 60. S. 466.

2. Arnd, Ueber die Durebgängigkeit der Darmwand eingeklemmter Brüche etc. Centralbl. f. Bakt. 1893. Bd. 13. S. 173.

3. Baisch, Bakteriologische und experimentelle Untersuchungen äber Cystitis nach gynäkologischen Operationen. Hegar's Beiträge. 1904. Bd. VIII. S. 297.

4. Barth, Zwei Fälle von Pyelitis gravid. Monatsschr. f. Geb. u. Gynäk. Bd. 26. S. 739.

5. Bietti, Typische Blennorrhoea neonatorum dureh Bact. coli cons. Klinische Monatsblätter f. Augenheilkunde. 1899.

6. Brentano, Die Ergebnisse bakteriologischer Bruchwasseruntersuchangen. Deutsche Zeitschr. f. Chir. 1896. No. 439.

7. Brucauff, Heilungsvorgänge bei Pyelonephritis. Virchow's Arch. 1901. Bd. 166.

8. Bumm, Zur Aetiologie des puerperalen Blasenkatarrhs. Verhandiung. d. deutschen Gesellsch. f. Gyn. 1886.

9. Burk, Untersuchungen über die Bakterien der Coligruppen. Centralbl. f. Bakt, 1907. No. 45.

10. Casper, a) Handbuch der Cystoskopie. 1898. b) Lehrbuch der Urologie. 1903.

11. Chrovstek-Egger, Zur Frage der Verwerthbarkeit bakteriologischer Harnbefunde etc. Wiener klin. Wochenschr. 1896. No. 30.

12. Cnopf, Ein casuistischer Beitrag zur Lehre von der Bakteriurie. Münch. med. Wochensehr. 1903. No. 40.

13. Deseniss, Ueber Pyelitis in der Schwangerschaft. Ref. Centralbl. f. Gyn. 1907. S. 986.

14. Doederlein, Die puerperalen Erkrankungen der Harnorgane. In Wincliel's Handb. der Geburtsh. 1906.

15. M c. Donald, Puerperalinfection. Ref. Centralbl. f. inn. Med. 28. S.301. 
16. v. Drigalski-Conradi, Ueber ein Verfahren zum Nachweis von Typhus:bakterien. Zeitschr. f. Hyg. 1902.

17. Edlefsen, Ueber die medicamentöse und diätetische Behandlung des Blasen- und Nierenbeckenkatarrhs. Deutsches Arch. f. kin. Med. Bd. 87.

18. Escherich-Pfauder, Das Bact. coli com. in Kolle-Wassermann's Handb. d. pathol. Mikroorg. Bd. II.

19. Faltin, a) Experimentelle Untersuchungen über die Infection der Harnblase. Centralbl. f. d. Krankh. d. Harn- u. Sexualorgane, 1901. H. 8 u. 9. - b) 86 Cystitiden. Ebenda. 1902. S.130. - c) Centralbl. f. Bakt. 1908 .

20. Fehling, Ueber Coliinfectionen. Münchener med. Woohenschr. Bd. 54. No. 27.

21. Finkelstein, Ueber Cystitis im Säuglingsalter. Jahrb.f. Kinderheilkunde. Bd. 43 , S. 148.

22. Frommel's Jahresbericht. 1908, 1909. Ref. Stumpf.

23. Gebhard, Das Bact. coli und seine Bedeutung für die Geburtshülfe. Zeitschr. f. Geb. u. Gyn. 1897. Bd. 37.

24. Goldberg, a) Ueber die culturelle Harnuntersuchungen otc. CentralbI. f. innere Med. Bd. 28. S. 393. - b) Ueber Bakteriurie. Centralbl. f. d. Krankh. der Harn- u. Sexualorgane. 1895. Bd. 4. S. 349.

25. Graf, Bakterienbefunde bei primärer Pyelonepbritis. Deutsche medic. Wochensehr. 1896. No. 38.

26. Günther, Bakteriologie. 6. Aufl. 1906.

27. Häberlin, Die acute idiapathische Pyelitis bei Schwangeren. Münchener med. Wochenschr. 1904.

28. Hofmeister, Ueber Organismen im Urin gesander Menschen. Fortschr. der Med. 1893.

29. Holle, Beiträge zur Frage der Durchgängigkeit ete. Centralbl. f. Bakt. 1907. Bd. 44. S. 325 .

30. Israel, Klinik der Nierenkrankheiten. 1901.

31. v. Kleckí, Bericht über die experimentellen Untersuchungen über den Durchtritt der Bakterien etc. Wiener klin. Wochenschr. 1907. No. 37. S. 1107.

32. Kolischer, Die Erkrankungen der weiblichen Harnröhre und Blase. 1898.

33. Kornfeld: a) Aetiologie und Klinik der Bakterinrie. 1906. - b) Ueber Colitoxämie. Ref. Wiener klin. Wochensohr. 1908. S. 342.

34. Krämer, Pyelitis gravidar. Ref. Deutsche medic. Wochenschr. 1907. S. 1845.

35. Krencker, Bact. coli com. als Sepsiserreger. Münchener med. Wochenschr. 1907 .

36. Küster, Die Chirurgie der Nieren etc. Deutsche Chirurgie. 52b. 1902.

37. Len hartz; Ueber die acute und chronische Nierenbeckenentzündung. Münchener med. Wockenschr. 1907.

38. Liebermeister, Ueber die Bedeutung des Bact. coli etc. Zeitschr. für llin. Med. 1906: Bd. 59.

39. Liewschitz, Beiträge zur Erkennung der Pyelitis. Dissert. Beilin 1906.

40. Mareus, Zur Frage der Durchgängigkeit des Darmes für: Bakterien. Wiener kin. Wochenschr. 1901.

41. Melohior, Cystitis und Urininfection. 1897, (Deutsche Uebersetzung.) 
42. Mirabeau, Schwangerschaftspyelitiden. Arch, f, Geb. und Gynäk, 1907. Bd. 82.

43. M. Neisser, Ein Fall von Mutation etc. Centralbl. für Bakt. Referate. Beiheft. S. 98.

44. Nen, Störungen der Nierenarbeit bei Parametrịtis. Med. Klinik. 1905. S. 1361 .

45. Oker-Blom, Beitr, zur Kenntniss des Eindringens des Bact. coli eto. Centralbl. f. Bakt. 1894. No. 15.

46. 01shausen, Verhandl. d. Gesellschaft für Geb. und Gynäk. Berlin 25. I. 1907.

47. Opitz: a) Beitr. zur Frage der Durchgängigkeit eto. Zeitschr. für Hyg. 1898. Bd. 29. - b) Die Pyelonephritis grav. et puerp. Zeitschr. f. Geb. und Gyn. 1905. Bd. 55.

48. Orlowski: a) Beitr. zur Kenntniss der biologischen und pathologischen Eigenschaft des Bact. col com. Ref. Centralbl. f. Bakt. 1897. S. 634. b) Ueber Pyelitis als Schwangerschaftscomplication. Monatsschr. f. Urologie. 1906. Xl.

49. Pawlowsky, Das Schicksal einiger pathogener Mikrorganismen etc. Zeitschr. für Hyg. 1909. Bd. 62.

50. Piltz, Ueber den Keimgehalt der Vulva und Urethra. Dieses Archiy Bd. 72. 1904.

51. Posner-Lewin, Untersuchangen über die Infection der Harnwege. Centralbl. f. d. Krankb. d. Harn- und Sexualorgane. 1896.

52. Posnẹr-Cohn, Ueber die Durchgängigkeit der Darmwand etc. Berliner klin. Wochenschr. 1900. No. 26.

53. Radziersky, Beitr. zur Kenntniss des Bact. coli. Zeitschr. f. Hyg. 1900. Bd. 34. S. 369.

54. Reed, Pyelonephritis in der Schwangersehaft. Ref. Centralbl. für Gyn. 1907. No. 35 .

55. Reymond, Ref. Centralbl. f. Bakt. 1894. XV.

56. Rolly, Zur Frage der Durchgängigkeit der. Niere. Münehener medic. Wochensehr. 1909. No. 37.

57. Rosenfeld, Zor Differentialdiagnose zwischen Cystitis und Pyelitis. Berliner klin. Wochensehr. 1898. S. 661.

58. Rovsing: a) Klinische und experimentelle Untersuchungen über die infectiösen Krankheiten dẹ Harnorgane. 1898. (Deutsche Uebersetzung.) b) Die Blasenentzündungen, ihre Aetiologie, Pathogenese und Behandlung. 1890. (Deutsche Uebersetzung.)

59. Ruppaner, Ueber Pyelonephritis in der Schwangerschaft. Münohener med. Wochenschr. 1906. S. 254.

60. Savor: a) Zur Aetiologie der acuten Pyelonephritis. Wiener klin. Wochenschr. 1894. No. 4 und 5. - b) Ueber den Keimgehalt der weiblichen Harnröhre. Beitr. z. Geb, u. Gyn. 1899. Bd. 2.

61. Scheidemantei, Ueber Pyelitis bei Frauen etc. Deutsche med. Wochenschr. 1908. S. 31.

62. Schenk-Austerlitz, Weitere Untersuchungen über den Keimgehalt der weiblichen Urethra. Wiener klin. Wochenschr. 1900. No. 14. 
63. Sehloffer, Bakteriologische Brachwasseruntersuchungen. Beitr. z. klin. Chirurg. 1895. XIV.

64. Schmidt, Untersuchungen über Hämolyse etc. Centralbl. f. Bakt. 1909. S. 359 .

65. Schmidt-Aschoff, Die Pyelonephritis in anatomischer und bakteriologischer Beziehung ete. 1897.

66. Sohumacher, Ein Beitrag zu der Lehre von der Pyelonephr. grav, et puerp. Dissert. Bonn 1905.

67. Sippe1, Pyonephrose, Pyelitis und Harnleitercompression. Centralbl. für Gyn. 1905.

68. Smith, Ceber Pyelonephritis in der Schwangersehaft etc. Ref. Centralbl. f. Gyn. 1905.

69. Stöckel: a) Die Erkrankungen d. weiblichen Harnorgane in Veit's Handb. der Gyn. 1907. - b) Zur Diagnose und Therapie der SchwangerschaftsPyelitis. Zeitschr. f. gynäk. Urolog. Bd. I. Heft 1.

70. Strassmann, Verhandl. der Gesellschaft für Geb. u Gyn. Berlin 14. 6. 1907.

71. Suter, Zur Aetiologie der infectiösen Erkrankungen der Harnwege. Zeitschr. f. Urologie. Bd. I.

72. Tanaka, Beitr. zur klinischen und bakteriologischen Untersuchung über Cystitis. Zeitschr. f. Urologie. 1909. III. Heft 5.

73. T'aussig, Ueber die postoperative Harnverhaltung und deren Folgen. Münchener med. Wochenschr. 1902. No. 40.

74. Thumim, Die Cystoskopie der Gynäkol. Volkmann's Samml. klin. Vorträge. 162/163.

75. Trumpp: a) Ueber Colicystitis im Kindesalter. Münchener med. Wochenschr. 1896. No. 42. - b) Jahrb. für Kinderheilk. 1897. Bd. 44. S. 268.

76. Uhlenhuth, Beitr. zur Pathogenität des Bact. coli com. Zeitschr. f. Hyg. 1897. Bd, 26.

77. Vogel, Der Verweilkatheter, seine Anwendung und Wirkungsweise. Berliner klin. Wochenschr. 1907.

78. Walthard, Ueber Bakterien in den Secreten der weiblichen Harnwege in Winckel's Handb. d. Geburtsh. Bd. III. 2.

79. Westhoff, Ueber eine Blasen-Darmfistel ete. Centralbl. f. Gyn. 1907. S. 1561.

80. Weindler, Beitr. zur Pyelitis grav. Gynäk. Rundschau. 1908. S. 772.

81. Weiss, Bakteriurie. Monatsschr. f. Geb. u. Gyn. Bd. 26. S. 844.

82. Winkler-Asch, Ascendirende Pyelitis. Wiener med. Wochenschr. Bd.57. S. 238.

83. Wreden, Zur Aetiologie der Cystitis. Centralbl. f. Chir. 1893.

84. v. Wunsehheim, Zur Aetiologie der Nephritis suppurat. Zeitschrift für Heilkunde. 1894. No. 15.

85. Wyssokowicz, Ueber die Passirbarkeit der kranken Nieren für Bakterien. Zeitschr. f. Hyg. 1908. Bd. 59.

86. Zickel, Beitarg zur Kenntniss der Pyelonephritis gravid. Monatssohr. f. Geb. u. Gyn. Bd. 23. H. 6.

87. Zuckerkandl in Nothnagel's spec. Path. u. Therapie. Bd. 19. 2, 2. 